\title{
Analytical and numerical calculation of the second-order moment of the beam using a capacitive pickup
}

\author{
Joel Alain Tsemo Kamga, Wolfgang F. O. Müller, and Thomas Weiland \\ Technische Universität Darmstadt, Institut für Theorie Elektromagnetischer Felder (TEMF), \\ Schlossgartenstraße 8, 64289 Darmstadt, Germany \\ (Received 24 November 2015; published 4 April 2016)
}

\begin{abstract}
Beam emittance has particular importance in particle physics, because it provides information about the quality of the particle beam. There are many techniques for measuring the beam emittance, such as that proposed by Miller et al. [Report No. SLAC-PUB-3186, (A) (1983)]. This technique is based on determining the emittance by measuring the second-order moment of the beam using quadrupole pickups consisting of four symmetrical electrodes placed around the beam pipe at $90^{\circ}$ intervals, respectively. Based on Miller's approach, two signal processing methods are generally used to get the quadrupole moment of the beam, namely the difference over sum and the log ratio [P. Li et al., IEEE Nuclear Science Symposium Conference Record, N24-404, 2007, pp. 1675-1678] methods. However, these traditional methods provide results with a good accuracy only for a well centered beam. The method presented in this paper, which starts with Miller's approach, considerably reduces the impact of the dipole signal on the quadrupole moment measurement for both small and large values of the beam position. Furthermore, a methodology for the numerical determination of the sensitivity of quadrupole pickups will be presented.
\end{abstract}

DOI: 10.1103/PhysRevAccelBeams.19.042801

\section{INTRODUCTION}

A capacitive or electrostatic pickup measures the charge collected on electrodes around the beam. It is usually used as a beam position monitor (BPM) in beam diagnostics for measuring the center of mass of the beam, whose longitudinal extent is much longer than the electrode length. However, using a certain combination of the signal voltages induced at the electrodes, the capacitive pickup can be operated as a quadrupole pickup. This pickup consists in general of four symmetric metallic electrodes placed in the same transverse plane and is sensitive to the second-order moment of an electrically charged particle beam, namely $\sigma_{x}^{2}-\sigma_{y}^{2}$. Here, $\sigma_{x}$ and $\sigma_{y}$ are the rms half beam dimensions in the transverse plane ( $x$ and $y$ directions).

Quadrupole pickups find application in several areas of accelerator physics, including in the measurement of the transverse beam emittance [1,2] and the detection of the injection mismatch causing envelope oscillations [3]. The emittance measurement technique was first suggested by Roger Miller et al. at SLAC National Accelerator Laboratory and requires six pickups, comprising four stripline electrodes respectively, placed at different positions in the linac [1]. Miller's technique is based on

*tsemo@temf.tu-darmstadt.de

Published by the American Physical Society under the terms of the Creative Commons Attribution 3.0 License. Further distribution of this work must maintain attribution to the author(s) and the published article's title, journal citation, and DOI. measuring the second-order moment of the electron beam at each of the six pickups and subsequently by means of the transfer matrix from a reference point in the linac to the pickup positions, determining the transverse beam emittances $\epsilon_{x}$ and $\epsilon_{y}$ in both transverse planes.

In synchrotrons, quadrupole pickups have also been used for measuring the beam emittance, thereunder the magnetic pickup design [2] comprising four antenna loop electrodes. The main advantage of this pickup model is its insensibility to the common mode signal [2].

According to the emittance measurement technique mentioned above and described in detail in [1], the most widely used signal processing methods for determining the quadrupole signal $\Xi$ (signal coming from a quadrupole pickup) and thus the second moment of the beam are as mentioned in the abstract the difference over sum and the $\log$ ratio method. It should be noted that $\Xi$, obtained with these methods, depends not only on $\sigma_{x}^{2}-\sigma_{y}^{2}$, which is the information we need, but also on the beam position $(\bar{x}, \bar{y})$,

$$
\Xi \propto \sigma_{x}^{2}-\sigma_{y}^{2}+\bar{x}^{2}-\bar{y}^{2} .
$$

Thus, to get $\sigma_{x}^{2}-\sigma_{y}^{2}$, the contribution of the beam displacement from the beam pipe center must first be subtracted from the quadrupole signal. However, to measure the beam position, it should be noted that the dipole signals are also sensitive to the sextupole moment of the beam, which can be neglected for small values of $\bar{x}$ and $\bar{y}$. For large values of $\bar{x}$ or $\bar{y}$, this is a significant effect and can lead to an inaccurate measurement of $\sigma_{x}^{2}-\sigma_{y}^{2}$. 
The main goal of this paper consists, starting from [1,4] and the log ratio method [5], in developing a pickup method that considerably decreases the beam position effect on the measurement of the quadrupole moment of the beam for both small and large values of $\bar{x}$ or $\bar{y}$. This method is called modified log ratio.

Furthermore, an asymmetrical BPM [6] installed at GSI SIS-18 will be investigated numerically using the electromagnetic field simulation softwares CST EMS and CST PS [7] to estimate its properties and evaluate its usage as a possible quadrupole pickup for the observation of beam size oscillations $[3,8]$.

SIS-18 [9] is a heavy ion synchrotron at GSI (Helmholtzzentrum für Schwerionenforschung) with a circumference of $216 \mathrm{~m}$, comprising 92 elements. In this ring accelerator, the ions are accelerated to an adjustable energy whose peak value depends on their mass-to-charge ratio [9]. The maximum magnetic rigidity of the synchrotron in SIS-18 is $18 \mathrm{Tm}$, thus explaining the number 18 in its name. Ions from the linear accelerator UNILAC can be accelerated in SIS18 to up to 90 percent of the speed of light $(270,000 \mathrm{~km} / \mathrm{s})$. The basic and technical parameters for SIS-18 can be found in [9] (page 8).

In Secs. II and III of this paper, analytical investigations will be performed, including the derivation of the modified $\log$ ratio method. The first part of Sec. IV will present the simulation results of the modified log ratio technique tested on the asymmetrical pickup mentioned above. Moreover, these results will be compared with those obtained with the traditional signal processing methods. In the second part of this section, the results arising from the simulation of the previously mentioned SIS-18 pickup model obtained in the frequency domain using the PIC solver of CST PS will be shown and compared with the simulation results coming from the electrostatic solver of CST EMS in terms of the pickup sensitivity to $\sigma_{x}^{2}-\sigma_{y}^{2}$. The last section of this paper is devoted to a summary and an outlook.

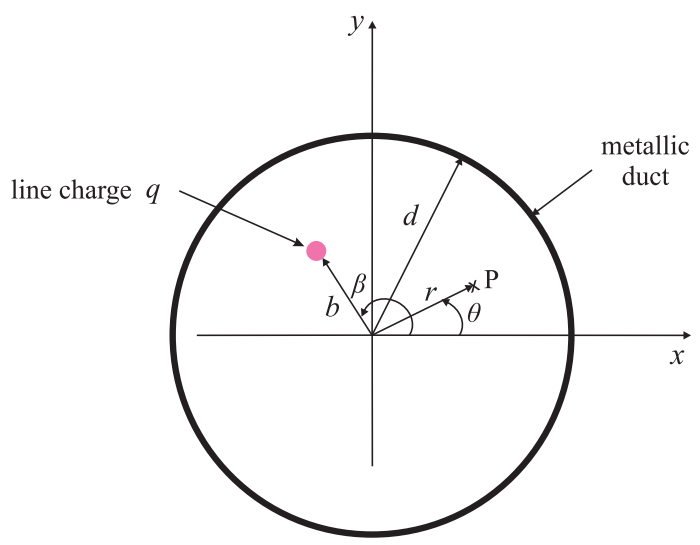

(a)

\section{SIGNAL VOLTAGES INDUCED AT THE PICKUP ELECTRODES}

\section{A. Electric field generated by a charged particle beam inside a metallic duct}

For bunches whose longitudinal extent is longer than the electrode length, the problem can be approximatively solved as a two-dimensional electrostatic problem.

In this case, the beam can be considered as an infinitely long line charge, and the electrostatic potential $V$ at any point $\mathrm{P}$ [see Fig. 1(a)] inside the beam pipe can be obtained using the method of image charge [10]:

$$
\begin{aligned}
V(r, \theta)= & \frac{q}{2 \pi \epsilon_{0}}\left[\ln \frac{1}{\sqrt{r^{2}+b^{2}-2 r b \cos (\theta-\beta)}}\right. \\
& \left.-\ln \frac{1}{\sqrt{r^{2}+\left(\frac{d^{2}}{b}\right)^{2}-\frac{2 r d^{2}}{b} \cos (\theta-\beta)}}+C\right] .
\end{aligned}
$$

The first term on the right side of Eq. (2) is the potential generated by the line charge and the second term is produced by its mirror charge. The constant $C=$ $\ln (b / d)$ has been determined using the boundary condition $[V(r=d, \theta)=0]$ at the surface of the grounded metallic duct. Assuming that $b$ is much less than the space point radius $r(b \ll r \sim d)$ and making use of the addition and subtraction theorems of the trigonometric functions, the above equation can be approximately expanded to the Taylor series as follows [10]:

$$
\begin{aligned}
V(r, \theta)= & \frac{q}{2 \pi \epsilon_{0}}\left[\ln \frac{d}{r}+\sum_{k=1}^{\infty} \frac{b^{k}}{k}\left(\frac{1}{r^{k}}-\frac{r^{k}}{d^{2 k}}\right)\right. \\
& \times \cos \{k(\theta-\beta)\}] .
\end{aligned}
$$

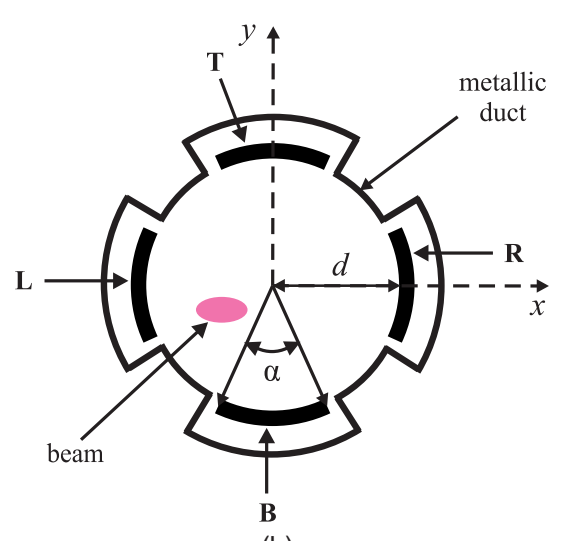

(b)

FIG. 1. (a) Line charge inside a metallic duct. (b) Cross section of the symmetrical four-electrodes pickup model used for calculations. 
Now, the electric field generated by the line charge inside a metallic beam pipe can be determined making use of the gradient of the potential $V$ given in Eq. (3):

$$
\vec{E}=-\nabla V
$$

Equation (4) can be expressed in the cylindrical coordinate system $(r, \theta, z)$ as [11]

$$
\vec{E}=-\left(\frac{\partial V}{\partial r} \vec{e}_{r}+\frac{1}{r} \frac{\partial V}{\partial \theta} \vec{e}_{\theta}+\frac{\partial V}{\partial z} \vec{e}_{z}\right) .
$$

From Eq. (5), the transverse components of the electric field vector can be obtained as follows:

$$
\begin{aligned}
E_{r}(r, \theta) & =-\frac{\partial V}{\partial r} \\
& =\frac{q}{2 \pi \epsilon_{0} r}\left[1+\sum_{k=1}^{\infty} b^{k}\left(\frac{1}{r^{k}}+\frac{r^{k}}{d^{2 k}}\right) \cos \{k(\theta-\beta)\}\right], \\
E_{\theta}(r, \theta) & =-\frac{1}{r} \frac{\partial V}{\partial \theta} \\
& =\frac{q}{2 \pi \epsilon_{0} r} \sum_{k=1}^{\infty} b^{k}\left(\frac{1}{r^{k}}-\frac{r^{k}}{d^{2 k}}\right) \sin \{k(\theta-\beta)\}
\end{aligned}
$$

\section{B. Signal voltages induced at the pickup electrodes}

The voltages induced by the beam charge on the electrodes will be derived using the pickup design depicted in Fig. 1(b), where R, L, T and B stand for the right, left, top and bottom electrodes, respectively. The angular width of each electrode is given by $\alpha$ and $d$ is the pickup radius.

The induced voltage $U$ at each electrode of the pickup model in Fig. 1(b) can be obtained from the area integration of the radial component $E_{r}(r=d)$ [given in Eq. (6b)] of the electric field on each plate, divided by the capacitance between the electrode and the beam pipe. Since the quadrupole signal to be calculated is a normalized signal to the sum of all electrode signals [see Eq. (11)] and due to the symmetry of the pickup design used for calculations, the capacitance between the plate and the beam pipe as well as the integration over the electrode length will be omitted in the derivation of the output voltages. For the right electrode $\mathrm{R}$, the output voltage is then given by

$$
U_{R} \propto d \int_{-\alpha / 2}^{\alpha / 2}\left(\varepsilon_{0} E_{r}\right) d \theta .
$$

In the case of a real beam, the line charge density $q$ in Fig. 1(a) may be multiplied by a suitable weighting function [12]. For Gaussian beam distributions in both transverse directions $\left(\sigma_{x}, \sigma_{y} \ll d\right)$ and by means of standard trigonometric identities $[\cos (\beta)=x / b$, $\sin (\beta)=y / b$ ], Eq. (7) becomes

$$
\begin{aligned}
U_{R} \propto & \frac{i_{b}}{2 \pi}\left[\alpha+4 \sin (\alpha / 2) \frac{\bar{x}}{d}\right. \\
& +2 \sin (\alpha)\left(\frac{\sigma_{x}^{2}-\sigma_{y}^{2}}{d^{2}}+\frac{\bar{x}^{2}-\bar{y}^{2}}{d^{2}}\right) \\
& +4 \frac{\sin (3 \alpha / 2)}{3}\left\{3\left(\frac{\sigma_{x}^{2}-\sigma_{y}^{2}}{d^{2}}\right)+\frac{\bar{x}^{2}-3 \bar{y}^{2}}{d^{2}}\right\} \frac{\bar{x}}{d} \\
& \left.+O\left(\frac{1}{d^{4}}\right)+\cdots\right],
\end{aligned}
$$

where $(\bar{x}, \bar{y})$ is the center of mass of the beam, $i_{b}$ the beam current and $O\left(1 / d^{4}\right)$ the fourth-order term. The output voltages at all electrodes are summarized below, where $U_{L}$, $U_{T}$ and $U_{B}$ have been derived analog to $U_{R}$ :

$$
\begin{aligned}
& U_{R} \propto \frac{i_{b}}{2 \pi} \alpha\left[1+z_{1 x}+z_{2}+z_{3 x}+\cdots\right], \\
& U_{L} \propto \frac{i_{b}}{2 \pi} \alpha\left[1-z_{1 x}+z_{2}-z_{3 x}+\cdots\right],
\end{aligned}
$$

$$
\begin{aligned}
& U_{T} \propto \frac{i_{b}}{2 \pi} \alpha\left[1+z_{1 y}-z_{2}-z_{3 y}+\cdots\right], \\
& U_{B} \propto \frac{i_{b}}{2 \pi} \alpha\left[1-z_{1 y}-z_{2}+z_{3 y}+\cdots\right] .
\end{aligned}
$$

Here $z_{1 x}, z_{1 y}, z_{2}, z_{3 x}$ and $z_{3 y}$ are the normalized first- (in both transverse directions), second- and third- (also in both transverse directions) order terms, respectively:

$$
\begin{aligned}
& z_{1 x}=4 \frac{\sin (\alpha / 2)}{\alpha} \frac{\bar{x}}{d}, \quad z_{1 y}=4 \frac{\sin (\alpha / 2)}{\alpha} \frac{\bar{y}}{d}, \\
& z_{2}=2 \frac{\sin (\alpha)}{\alpha}\left(\frac{\sigma_{x}^{2}-\sigma_{y}^{2}}{d^{2}}+\frac{\bar{x}^{2}-\bar{y}^{2}}{d^{2}}\right), \\
& z_{3 x}=4 \frac{\sin (3 \alpha / 2)}{3 \alpha}\left\{3\left(\frac{\sigma_{x}^{2}-\sigma_{y}^{2}}{d^{2}}\right)+\frac{\bar{x}^{2}-3 \bar{y}^{2}}{d^{2}}\right\} \frac{\bar{x}}{d}, \\
& z_{3 y}=4 \frac{\sin (3 \alpha / 2)}{3 \alpha}\left\{3\left(\frac{\sigma_{x}^{2}-\sigma_{y}^{2}}{d^{2}}\right)+\frac{3 \bar{x}^{2}-\bar{y}^{2}}{d^{2}}\right\} \frac{\bar{y}}{d} .
\end{aligned}
$$

Since we are interested in the second-order moment of the beam, the multipole expansion is limited here to the sextupole term. 


\section{METHODS TO PICK UP THE QUADRUPOLE MOMENT OF THE BEAM}

\section{A. Traditional methods}

\section{Difference over sum}

Looking at the output voltages given in Eqs. (9a), (9b), (9c) and (9d), it is obvious that the most simple approach to obtain $z_{2}$ is to apply the following signal combination: $\left(U_{R}+U_{L}\right)-\left(U_{T}+U_{B}\right)$. To make the resulting signal independent of the beam current, the signal combination mentioned above has to be divided by the sum of all electrode signals; this is why this method is called difference over sum:

$$
\begin{aligned}
\Xi_{\Delta / \Sigma} & =\frac{\left(U_{R}+U_{L}\right)-\left(U_{T}+U_{B}\right)}{U_{R}+U_{L}+U_{T}+U_{B}} \\
& =z_{2} \\
& =\underbrace{\frac{\sin (\alpha)}{\alpha} \frac{1}{d^{2}}}_{S_{\Delta / \Sigma}}\left(\sigma_{x}^{2}-\sigma_{y}^{2}+\bar{x}^{2}-\bar{y}^{2}\right),
\end{aligned}
$$

where $\Xi_{\Delta / \Sigma}$ is called the quadrupole signal and $S_{\Delta / \Sigma}$ the sensitivity of the quadrupole pickup illustrated in Fig. 1(b). The index $\Delta / \Sigma$ is related to the name of the method.

To obtain $\sigma_{x}^{2}-\sigma_{y}^{2}$ from Eq. (12), the beam position must be known. This can be obtained from the horizontal and vertical dipole signals $\Delta_{x}$ and $\Delta_{y}$ :

$$
\begin{aligned}
\Delta_{x} & =\frac{U_{R}-U_{L}}{U_{R}+U_{L}} \\
& =z_{1 x}+z_{3 x} \\
& =\underbrace{4 \frac{\sin (\alpha / 2)}{\alpha} \frac{1}{d}}_{S_{d}} \bar{x}+z_{3 x},
\end{aligned}
$$

where $S_{d}$ stands for the sensitivity of the beam position monitor. Since the pickup design in Fig. 1(b) is symmetrical, Eq. (14) also holds for the vertical dipole signal by replacing $\bar{x}$ and $z_{3 x}$ with $\bar{y}$ and $-z_{3 y}$, respectively. From Eq. (14), while omitting the third-order term $z_{3 x}$, respectively $z_{3 y}$, one can approximately solve $\bar{x}$, respectively $\bar{y}$, and combine the result into Eq. (12). The second moment can then be represented as follows:

$$
\sigma_{x}^{2}-\sigma_{y}^{2}=\frac{\Xi_{\Delta / \Sigma}}{S_{\Delta / \Sigma}}-\left[\left(\frac{\Delta_{x}}{S_{d}}\right)^{2}-\left(\frac{\Delta_{y}}{S_{d}}\right)^{2}\right] .
$$

More details about this method can be found in $[1,4]$.

\section{Log ratio}

Using a logarithmic amplifier at the output of each electrode, and assuming that the sum of all multipole terms starting from the first-order term is less than the monopole term, then the quadrupole signal $\Xi_{\log }$ is given by [5]

$$
\begin{aligned}
\Xi_{\log }= & 20 \log \left(\frac{U_{R} \cdot U_{L}}{U_{T} \cdot U_{B}}\right) \\
= & 20 \log \left(U_{R}\right)+20 \log \left(U_{L}\right)-20 \log \left(U_{T}\right) \\
& -20 \log \left(U_{B}\right) \\
= & \frac{80}{\ln (10)}\left[z_{2}-\frac{z_{1 x}^{2}-z_{1 y}^{2}}{4}\right]+O\left(\frac{1}{d^{4}}\right) \\
= & \frac{160}{\ln (10)} \frac{\sin (\alpha)}{\alpha}[\frac{\sigma_{x}^{2}-\sigma_{y}^{2}}{d^{2}}+\underbrace{\left(1-\frac{\tan (\alpha / 2)}{\alpha}\right)}_{m} \frac{\bar{x}^{2}-\bar{y}^{2}}{d^{2}}] \\
& +O\left(\frac{1}{d^{4}}\right)
\end{aligned}
$$

In the same way as in the previous section, the second moment of the beam can be obtained as follows:

$$
\sigma_{x}^{2}-\sigma_{y}^{2}=\frac{\Xi_{\log }}{S_{\log }}-m\left[\left(\frac{\Delta_{x, \log }}{S_{d, \log }}\right)^{2}-\left(\frac{\Delta_{y, \log }}{S_{d, \log }}\right)^{2}\right]
$$

with

$$
\begin{aligned}
S_{\log } & =\frac{160}{\ln (10)} \frac{1}{d^{2}} \frac{\sin (\alpha)}{\alpha}\left(\text { in } \mathrm{dB} / \mathrm{mm}^{2}\right) \\
\Delta_{x, \log } & =20 \log \left(U_{R}\right)-20 \log \left(U_{L}\right) \\
& =\underbrace{\frac{160}{\ln (10)} \frac{1}{d} \frac{\sin (\alpha / 2)}{\alpha}}_{S_{d, \log }} \bar{x}+O\left(\frac{1}{d^{3}}\right) \\
\Delta_{y, \log } & =20 \log \left(U_{T}\right)-20 \log \left(U_{B}\right) \\
& =\underbrace{\frac{160}{\ln (10)} \frac{1}{d} \frac{\sin (\alpha / 2)}{\alpha}}_{S_{d, \log }} \bar{y}+O\left(\frac{1}{d^{3}}\right) .
\end{aligned}
$$

More details about the derivation and work principle of the $\log$ ratio can be found in $[5,13]$.

\section{B. Modified log ratio method}

\section{Derivation of $\sigma_{x}^{2}-\sigma_{y}^{2}$}

Looking at Eq. (18), it is obvious that, for all values of the angular electrode width $\alpha$, the term $m$ is less than or equal to 0.5. The aim of this method is to find an approach to have the term $m$ in Eq. (18) equal zero for any angular electrode width $\alpha$. Looking at Eq. (17), one can state that the dipole terms $z_{1 x}$ and $z_{1 y}$ generate the factor $m$ through the logarithm development $\ln \left(1+z_{1 x, y}+z_{2}+\cdots\right)$ (see more details in [5]). Going away from there, $z_{1 x}^{2}$ and $z_{1 y}^{2}$ 
in Eq. (17) will be modified such that the new resultant term $m$ vanishes. The first step of the method consists in adding a weighted dipole signal to each electrode output signal,

$$
\begin{aligned}
U_{R, \text { mod }}= & U_{R}+c_{x} \cdot\left(U_{R}-U_{L}\right) \\
= & \frac{i_{b}}{2 \pi} \alpha\left[1+z_{1 x}+z_{2}+z_{3 x}+\mathbf{2}_{\mathbf{x}}\left(\mathbf{z}_{1 \mathbf{x}}+\mathbf{z}_{\mathbf{3 x}}\right)+\cdots\right] \\
= & \frac{i_{b}}{2 \pi} \alpha\left[1+\left(1+2 c_{x}\right) z_{1 x}+z_{2}\right. \\
& \left.+\left(1+2 c_{x}\right) z_{3 x}+\cdots\right], \\
U_{L, \text { mod }}= & U_{L}-c_{x} \cdot\left(U_{R}-U_{L}\right) \\
= & \frac{i_{b}}{2 \pi} \alpha\left[1-z_{1 x}+z_{2}-z_{3 x}-\mathbf{2} \mathbf{c}_{\mathbf{x}}\left(\mathbf{z}_{\mathbf{1 x}}+\mathbf{z}_{\mathbf{3 x}}\right)+\cdots\right] \\
= & \frac{i_{b}}{2 \pi} \alpha\left[1-\left(1+2 c_{x}\right) z_{1 x}+z_{2}\right. \\
& \left.-\left(1+2 c_{x}\right) z_{3 x}+\cdots\right], \\
U_{T, \text { mod }}= & U_{T}+c_{y} \cdot\left(U_{T}-U_{B}\right) \\
= & \frac{i_{b}}{2 \pi} \alpha\left[1+z_{1 y}-z_{2}-z_{3 y}+\mathbf{2} \mathbf{c}_{\mathbf{y}}\left(\mathbf{z}_{\mathbf{1 y}}-\mathbf{z}_{\mathbf{3 y}}\right)+\cdots\right] \\
= & \frac{i_{b}}{2 \pi} \alpha\left[1+\left(1+2 c_{y}\right) z_{1 y}-z_{2}\right. \\
& \left.-\left(1+2 c_{y}\right) z_{3 y}+\cdots\right], \\
U_{B, \text { mod }}= & U_{B}-c_{y} \cdot\left(U_{T}-U_{B}\right) \\
= & \frac{i_{b}}{2 \pi} \alpha\left[1-z_{1 y}-z_{2}+z_{3 y}-\mathbf{2} \mathbf{c}_{\mathbf{y}}\left(\mathbf{z}_{\mathbf{1 y}}-\mathbf{z}_{\mathbf{3 y}}\right)+\cdots\right] \\
= & \frac{i_{b}}{2 \pi} \alpha\left[1-\left(1+2 c_{y}\right) z_{1 y}-z_{2}\right. \\
& \left.+\left(1+2 c_{y}\right) z_{3 y}+\cdots\right], \\
&
\end{aligned}
$$

where $c_{x}$ and $c_{y}$ are the weighting factors of the weighted dipole signals in the horizontal and vertical planes, respectively. The terms, marked in bold in the above equations, are the added weighted dipole signals. It should be noted that, for example, the weighted dipole signal $c_{x}\left(U_{R}-U_{L}\right)$, added to the output signal of the right electrode $R$, must be subtracted from the output signal of the left electrode L, so that later by carrying out the signal combination for obtaining the quadrupole signal the monopole and transverse dipole terms still remain suppressed.

The next step of this technique consists in applying a logarithmic amplifier to the signals given in Eqs. (21a), (21b), (21c) and (21d). After that, the quadrupole signal $\Xi_{\text {mod }}$ can be obtained analogously to that of the log ratio method, which was in the previous section:

$$
\begin{aligned}
\Xi_{\text {mod }}= & 20 \log \left(\frac{U_{R, \text { mod }} \cdot U_{L, \text { mod }}}{U_{T, \text { mod }} \cdot U_{B, \text { mod }}}\right) \\
= & \frac{80}{\ln (10)}\left[z_{2}-\frac{\left(1+2 c_{x}\right)^{2} z_{1 x}^{2}-\left(1+2 c_{y}\right)^{2} z_{1 y}^{2}}{4}\right] \\
& +O\left(\frac{1}{d^{4}}, c_{x}, c_{y}\right) \\
= & \frac{160}{\ln (10)} \frac{\sin (\alpha)}{\alpha}\left[\frac{\sigma_{x}^{2}-\sigma_{y}^{2}}{d^{2}}+m_{x} \frac{\bar{x}^{2}}{d^{2}}-m_{y} \frac{\bar{y}^{2}}{d^{2}}\right] \\
& +O\left(\frac{1}{d^{4}}, c_{x}, c_{y}\right),
\end{aligned}
$$

with

$$
\begin{aligned}
& m_{x}=1-\left(1+2 c_{x}\right)^{2} \frac{\tan (\alpha / 2)}{\alpha}, \\
& m_{y}=1-\left(1+2 c_{y}\right)^{2} \frac{\tan (\alpha / 2)}{\alpha} .
\end{aligned}
$$

More details about the derivation of $\Xi_{\text {mod }}$ can be found in Appendix A 1 or in [14] (page 62).

The weighting factors $c_{x}$ and $c_{y}$ can now be determined as the roots of the terms $m_{x}$ and $m_{y}$ :

$$
\begin{aligned}
& m_{x}=0 \Rightarrow c_{x}=-\frac{1}{2}\left(1 \mp \sqrt{\frac{\alpha}{\tan (\alpha / 2)}}\right), \\
& m_{y}=0 \Rightarrow c_{y}=-\frac{1}{2}\left(1 \mp \sqrt{\frac{\alpha}{\tan (\alpha / 2)}}\right) .
\end{aligned}
$$

From Eqs. (26a) and (26b), it is obvious that the factors $c_{x}$ and $c_{y}$ are equal to each other as expected, because the pickup design used for the analytical investigations is symmetrical with respect to the $x$ axis and to the $y$ axis [see Fig. 1(b)]. For an asymmetrical pickup, where the angular width of all electrodes or the pickup radius $d$ in both planes is not the same, $c_{x}$ will be unequal to $c_{y}$.

Now, substituting Eqs. (26a) and (26b) into Eq. (24) and neglecting the fourth-order term $O\left(1 / d^{4}, c_{x}, c_{y}\right)$ in Eq. (24), the quadrupole moment can be approximatively obtained as

$$
\sigma_{x}^{2}-\sigma_{y}^{2}=\frac{\Xi_{\mathrm{mod}}}{S_{\log }}
$$

where $S_{\log }$ is the sensitivity of the quadrupole pickup according to the log ratio method. A graphical representation of the modified log ratio method is illustrated in Fig. 2.

In Fig. 3, the quadrupole moment of the beam obtained according to Eqs. (15), (19) and (27) is depicted as a function of the horizontal and vertical beam position. The 


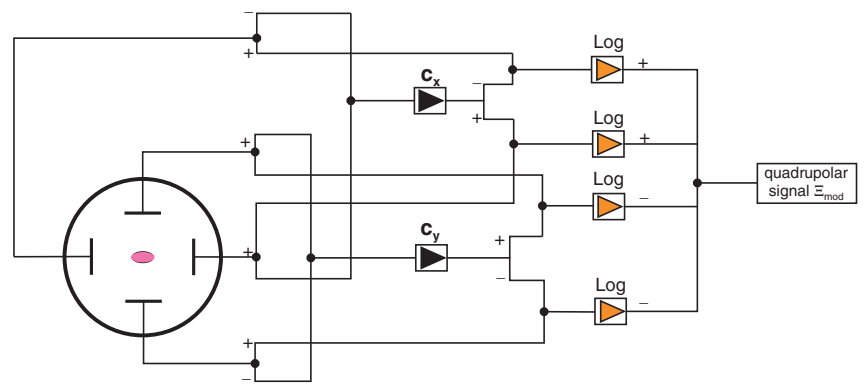

FIG. 2. Graphical representation of the modified log ratio technique.

factors $c_{x}=c_{y}=0.1885$ have been obtained according to Eqs. (26a) and (26b). Looking at the upper graph in Fig. 3, one can see that in the range of $\bar{x}$ between 0 and around $10 \%$ of the pickup radius $d$, the quadrupole moment provided by the three methods is almost the same and equal to the ideal value (setting value) of $\left(\sigma_{x}^{2}-\sigma_{y}^{2}\right) / d^{2}$. This can be explained by the fact that in the mentioned range of $\bar{x}$ and $\bar{y}$ the contribution of the sextupole moment in the dipole signal in both horizontal and vertical planes is too small and can be neglected. Thus, the dipole signal in that $\bar{x}, \bar{y}$ range $(0 \cdots 0.1 d)$ can be considered as linear to the beam position, as can be seen in Fig. 4 .

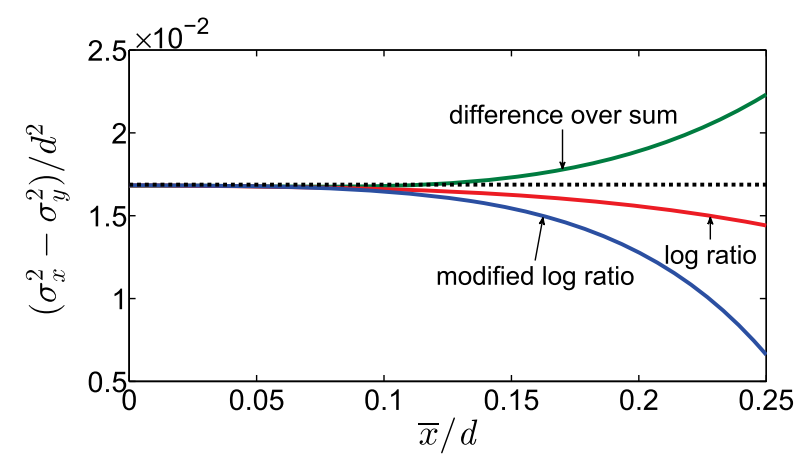

(a)

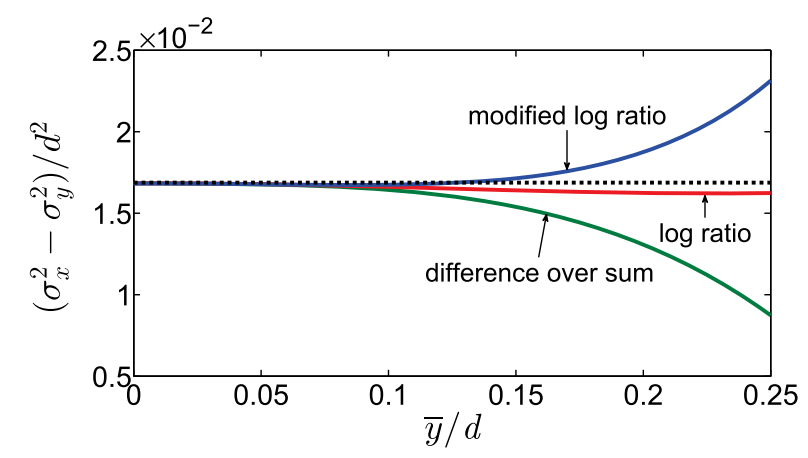

(b)

FIG. 3. Normalized quadrupole moment $\left(\sigma_{x}^{2}-\sigma_{y}^{2}\right) / d^{2}$ as a function of the beam position; $\sigma_{x} / d=0.15, \sigma_{y} / d=0.075$, $\alpha=45^{\circ}, \quad c_{x}=c_{y}=0.1885$ : (a) as a function of $\bar{x} / d$, $\bar{y} / d=0.05$; (b) as a function of $\bar{y} / d, \bar{x} / d=0.05$.
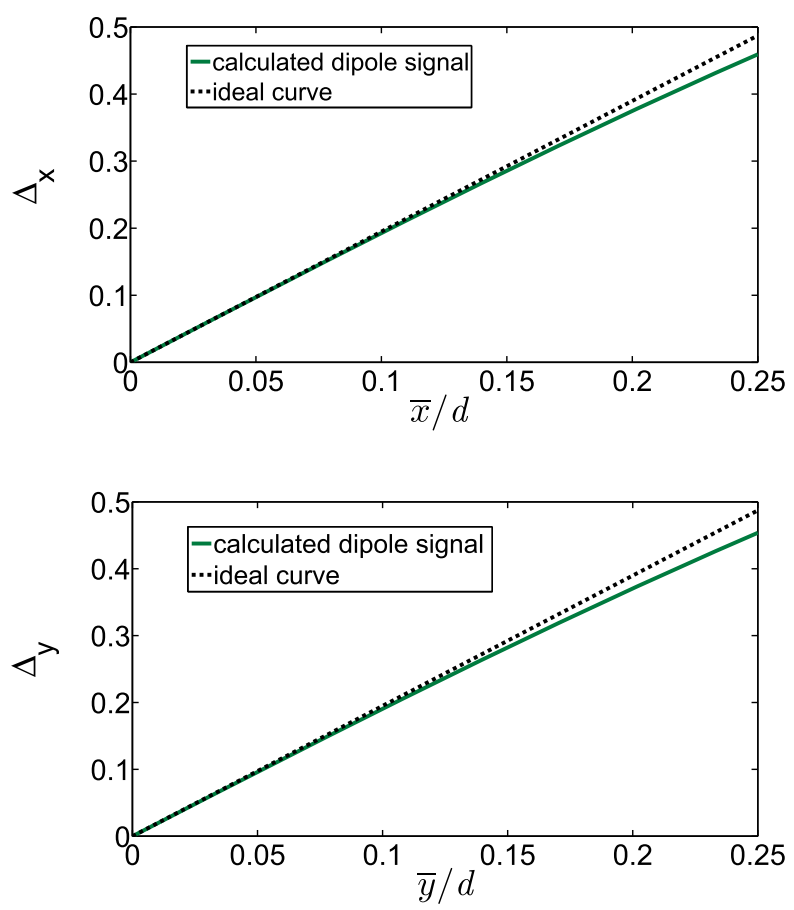

FIG. 4. Above: $\Delta_{x}$ as a function of $\bar{x} / d, \bar{y} / d=0.05$; below: $\Delta_{y}$ as a function of $\bar{y} / d, \bar{x} / d=0.05$.

The horizontal and vertical dipole signals $\Delta_{x}$ and $\Delta_{y}$ are obtained using the difference over sum method according to Eq. (14). In Fig. 4, the ideal curve represents the dipole signal, which is linear with respect to the beam position and corresponds in this case to the terms $z_{1 x}$ and $z_{1 y}$ for the horizontal and vertical planes, respectively. These terms are given in Eq. (14).

For a value of $\bar{x} / d$ greater than 0.1 , however, the traditional methods provide slightly better results as one can see on the upper diagram in Fig. 3. But, by means of appropriate values of the weighting factors $c_{x}$ and $c_{y}$, the modified log ratio method can be optimized as will be shown in the second part of this section.

\section{Optimization of the method}

In this section, the modified log ratio will be optimized by matching the factors $c_{x}$ and $c_{y}$. To better understand the matching approach, the quadrupole signal according to Eq. (24) can be rewritten as follows:

$$
\Xi_{\mathrm{mod}}=\frac{160}{\ln (10)} \frac{\sin (\alpha)}{\alpha}\left[\frac{\sigma_{x}^{2}-\sigma_{y}^{2}}{d^{2}}+P\right]
$$

with

$$
P=m_{x} \frac{\bar{x}^{2}}{d^{2}}-m_{y} \frac{\bar{y}^{2}}{d^{2}}+O\left(\frac{1}{d^{4}}, c_{x}, c_{y}\right) .
$$

This equation shows that the fourth-order term, which is detailed in Appendix A 1 and in [14] (page 62), also 
depends on $c_{x}$ and $c_{y}$. The main idea of the optimization consists in calculating the weighting factors for each value of $\bar{x}$ and $\bar{y}$ such that the term $P$ in the above equation becomes zero. The optimized horizontal, respectively the vertical damping factor can then be set as the arithmetic mean of all $c_{x}(\bar{x})$ respectively $c_{y}(\bar{y})$. It should be noted that to calculate $c_{x}(\bar{x})$, the vertical beam position $\bar{y}$ should be set to zero and vice versa for determining $c_{y}(\bar{y})$.

For the optimization of the modified log ratio method, both a fixed-beam dimension as well as a range of the beam position should be defined in which the weighting factors will be optimized. It should be assumed that $\bar{x} / d$ and $\bar{y} / d$ vary between 0 and 0.15 and the beam has the transverse dimension $\sigma_{x} / d=0.15, \sigma_{y} / d=0.075$. Since in the $\bar{y} / d$ range up to 0.15 , the quadrupole moment obtained with this method is almost constant [see the blue curve in Fig. 3(b)], therefore only $c_{x}$ will be optimized and this will occur in the $\bar{x} / d$ range from 0.1 to 0.15 . Figure 5 shows for $\bar{y}=0$ the values of $c_{x}$ for 21 equidistant $\bar{x} / d$ values from 0.1 to 0.15 , such that the term $P$ in Eq. (29) vanishes. In other words, for each value of $\bar{x} / d$ the factor $c_{x}$ was determined as a root of the function $P$.

The optimized factor $c_{x}=0.1735$ was obtained as the arithmetical mean of all weighting factors illustrated in Fig. 5. To calculate the optimized value of $c_{x}$, the arithmetic average has been used as an example. Nevertheless, depending on the optimization wishes, other fitting techniques can be used, which can lead to better results. Figure 6 shows the normalized quadrupole moment calculated with the modified log ratio method for once a not optimized and once an optimized amplification factor $c_{x}$ as a function of the horizontal beam position. The black dotted line in that figure stands for the ideal value of $\left(\sigma_{x}^{2}-\sigma_{y}^{2}\right) / d^{2}$, which refers to the setting value of $\left(\sigma_{x}^{2}-\sigma_{y}^{2}\right) / d^{2}$ used for calculations.

From Fig. 6 it is obvious that the quadrupole moment calculated using the optimized amplification coefficient $c_{x}=0.1735$ is less dependent on the beam position. Nevertheless, it should be noted that $c_{x}=0.1735$ was determined for one transverse beam size $\left(\sigma_{x} / d=0.15\right.$, $\left.\sigma_{y} / d=0.075\right)$. Now, taking into account that the term $P$ in

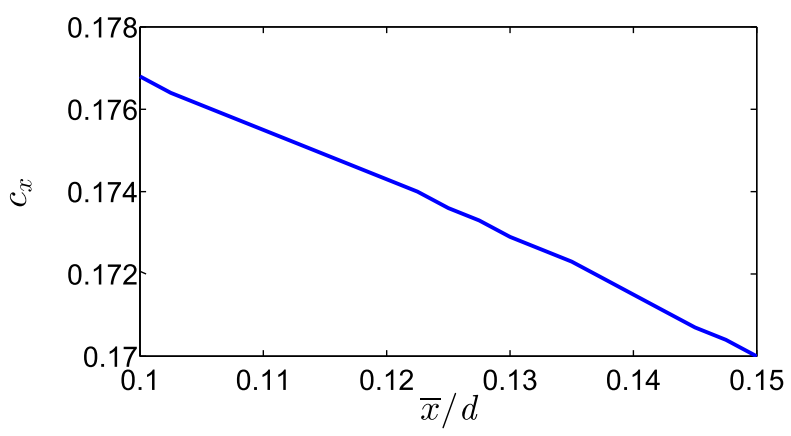

FIG. 5. Weighting factor $c_{x}$ as a function of $\bar{x} / d ; c_{y}=0.1885$, $\sigma_{x} / d=0.15, \sigma_{y} / d=0.075, \bar{y}=0$.

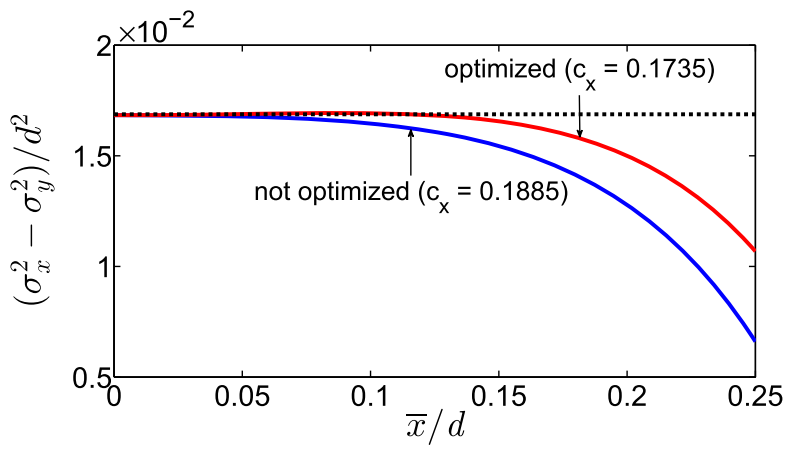

FIG. 6. $\left(\sigma_{x}^{2}-\sigma_{y}^{2}\right) / d^{2}$ as a function of $\bar{x} / d$ for a not and an optimized factor $c_{x} ; c_{y}=0.1885$.

Eq. (28) also depends on the transverse beam size through the fourth-order term, the quadrupole moment should be also calculated for different values of $\sigma_{x}$ using the same optimized value of $c_{x}$. Since the curves of the second moment as a function of the beam size are so close that the difference between them is not clear, Fig. 7 shows instead only their relative deviations from the ideal value. Here, it should be noted that this relative deviation is equal to the absolute deviation divided by the maximal value of the ideal curve. The quadrupole moment was calculated for a variation of $\sigma_{x} / d$ in the range of 0.075 to 0.3 with a constant $\sigma_{y} / d=0.075$ and constant beam position.

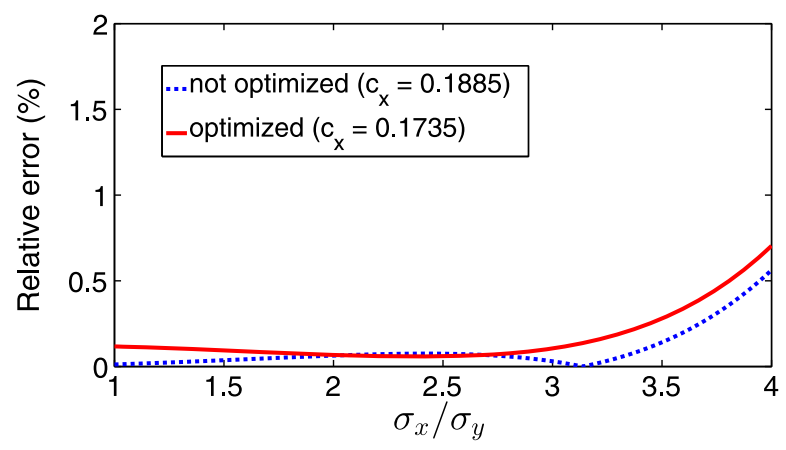

(a)

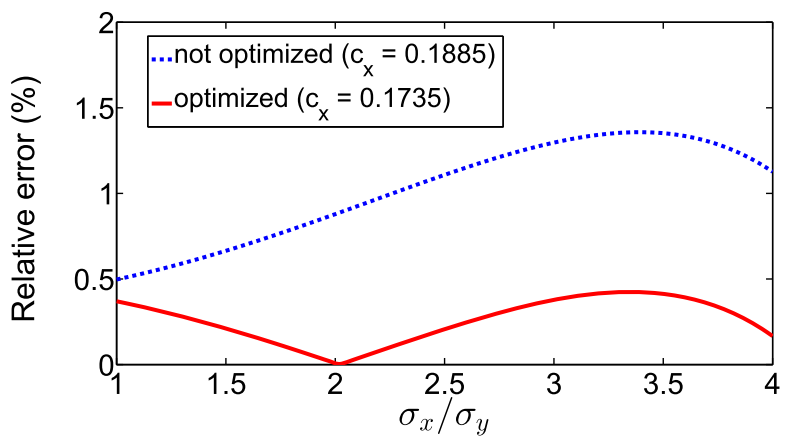

(b)

FIG. 7. Relative error of the calculated values of $\left(\sigma_{x}^{2}-\sigma_{y}^{2}\right) / d^{2}$ as a function of $\sigma_{x} / \sigma_{y}, \sigma_{y} / d=0.075$ : (a) $\bar{x} / d=0.05, \bar{y}=0$; (b) $\bar{x} / d=0.125, \bar{y}=0$. 
Figure 7(a) shows an insignificant difference between both curves due to the small value of the used beam position $(\bar{x} / d=0.05, \bar{y}=0)$. In contrast, in Fig. 7(b) one can see that the curve with $c_{x}=0.1735$ provides a better result over the whole range of the used beam sizes. Based on these results, one can conclude that the amplification factor $c_{x}=0.1735$ obtained for $\sigma_{x} / d=0.15, \sigma_{y} / d=$ 0.075 can also be applied to the other beam sizes plotted in Fig. 7. Therefore, this factor will be used in the following as the horizontal amplification factor of the modified log ratio method.

Figure 9 shows for all pickup methods the quadrupole moment, calculated according to Eqs. (15), (19) and (27) as a function of the beam position. The setting values of the beam size used for calculations are $\sigma_{x} / d=0.15$ and $\sigma_{y} / d=0.075$, which lead to the ideal value (setting value) of the normalized quadrupole moment $\left(\sigma_{x}^{2}-\sigma_{y}^{2}\right) / d^{2}=$ $1.6875 \times 10^{-2}$. This is represented in the figure by the black dotted line. Looking at all images in Fig. 9, it is obvious that in the whole range of $\bar{x} / d$ until 0.15 the modified log ratio is the method with the lowest dependence on the beam position. In other words, one can say that, for a beam located inside a quadrant bounded by the planes $\bar{x}=0, \bar{x} / d=0.15, \bar{y}=0$ and $\bar{y} / d=0.15$, the modified $\log$ ratio method is less dependent on the beam position. To get a better overview of the difference between the three

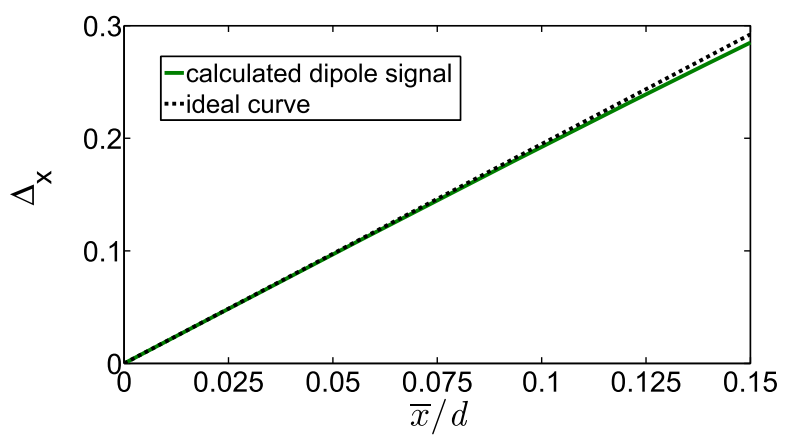

(a) $\bar{y} / d=0.15$

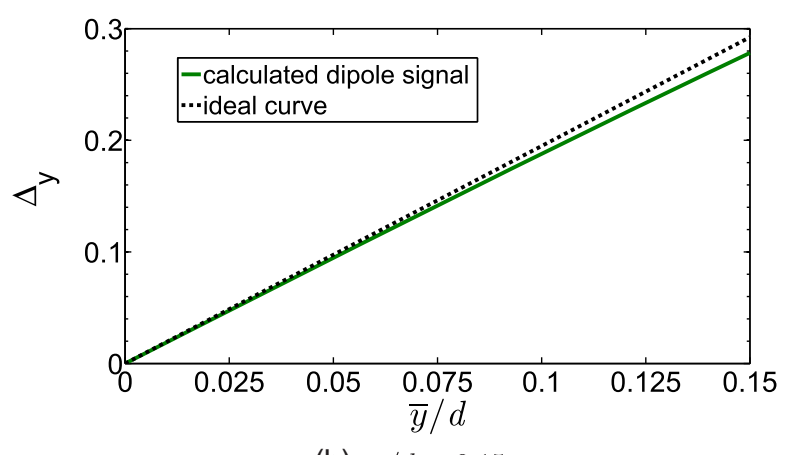

(b) $\bar{x} / d=0.15$

FIG. 8. Dipole signals calculated with the difference over sum method; $\sigma_{x} / d=0.15, \sigma_{y} / d=0.075$. methods, the relative deviation of the calculated values of $\left(\sigma_{x}^{2}-\sigma_{y}^{2}\right) / d^{2}$ from its ideal value is illustrated in Fig. 10 for $\bar{y} / d=0.125$ and $\bar{y} / d=0.15$. The reason for the large deviation in the conventional methods lies in the dipole signals, since these (dipole signals) depend not only on the beam position, but also on the sextupole terms $z_{3 x}$ and $z_{3 y}$ [see Eq. (14)]. For large values $\bar{x}$ or $\bar{y}$, this leads to a nonlinearity of the dipole signals with respect to the position as one can see below in Fig. 8. Figure 8(a) shows the horizontal dipole signal $\Delta_{x}$ as a function of $\bar{x} / d$ for a fixed value of $\bar{y} / d$ while Fig. 8(b) shows the vertical dipole signal $\Delta_{y}$ as a function of $\bar{y} / d$ for a fixed value of $\bar{x} / d$. The $\bar{x} / d$ range $(0.1 \leq \bar{x} / d \leq 0.15)$ chosen here for the optimization was just an example to show how to optimize the modified log ratio method when the interval of the beam position is known. In Sec. IV, a real beam position monitor model installed at GSI SIS-18 will be simulated as a quadrupole pickup. Since for the SIS-18 operation the variation of the beam position can be estimated, it will be easy to optimize the method presented in this paper.

\section{SIMULATION RESULTS}

The pickup model simulated in this paper is, as mentioned at the end of the previous section, a beam position monitor installed at GSI SIS-18. Since for typical SIS-18 operation the bunch is long compared to the pickup electrodes, the numerical investigation of the modified $\log$ ratio and the traditional methods were carried out using the electrostatic solver of CST EMS, and the results are presented in the first part of this section. The second part of this section will show the simulation results in the frequency domain carried out using the PIC solver of CST PS, and these will be compared with the results from the electrostatic solver in terms of the quadrupole signal and pickup sensitivity. The figure below shows the front view and the dimensions of the simulated pickup design. R, T, L and B in Fig. 11(a) represent the right, top, left and bottom electrodes, respectively.

\section{A. Simulations with the electrostatic solver of CST EMS}

\section{Pickup's sensitivity}

Since the pickup depicted in Fig. 11 has been operated until now as a beam position monitor for measuring the center of mass of the beam, the first thing to do is to check if the pickup is sensitive to the quadrupole signal defined in Eq. (1). Some simulations have been performed for this to calculate the quadrupole signal $\Xi$ once for a centered beam $(\bar{x}=\bar{y}=0)$ as a function of the beam size $\left(\sigma_{x}, \sigma_{y}\right)$ and once for a round beam $\left(\sigma_{x}=\sigma_{y}=7.5 \mathrm{~mm}\right)$ as a function of the beam position and the results are shown in Fig. 12. The beam was modeled by a perfect conductive elliptical cylinder [the red cylinder in Fig. 11(b)] of radii $\left(\sigma_{x}, \sigma_{y}\right)$. For the excitation, a potential of $1 \mathrm{~V}$ was applied to the 


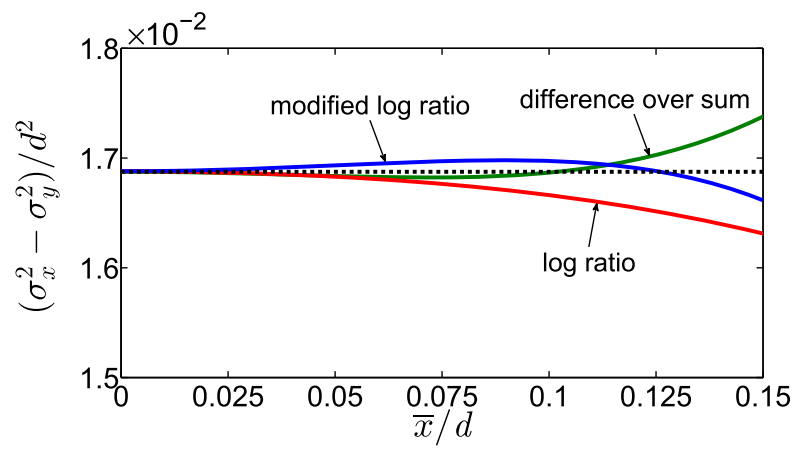

(a) $\bar{y}=0$

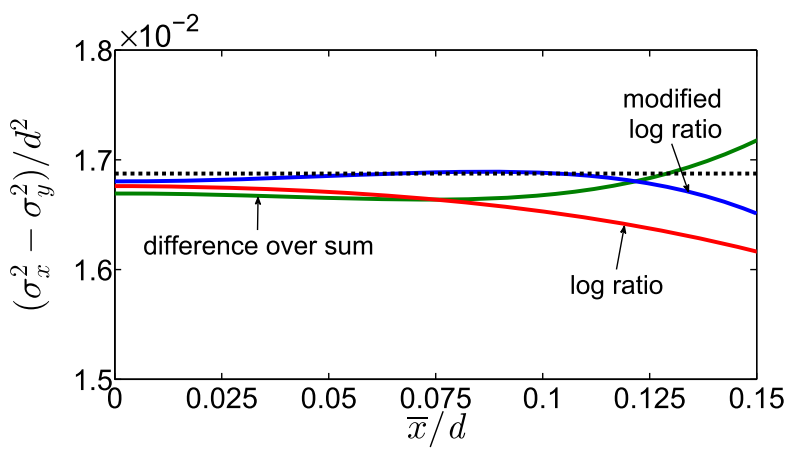

(c) $\bar{y} / d=0.075$

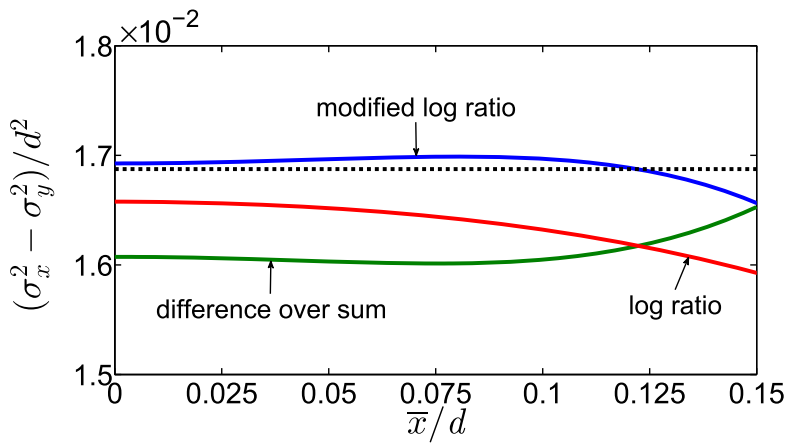

(e) $\bar{y} / d=0.125$

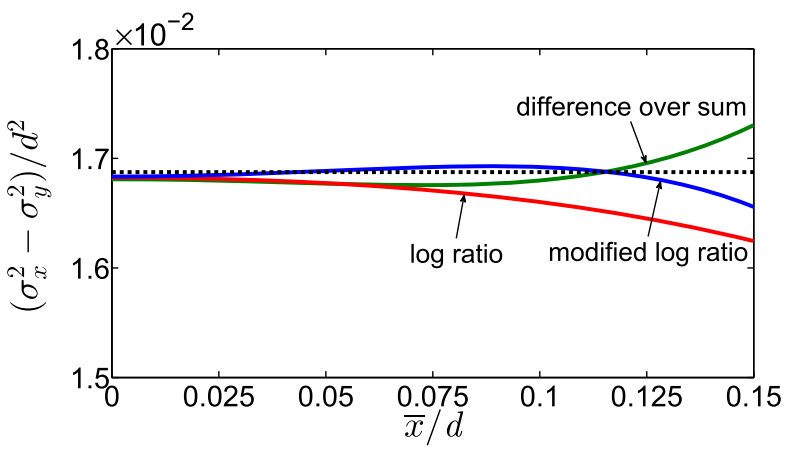

(b) $\bar{y} / d=0.05$

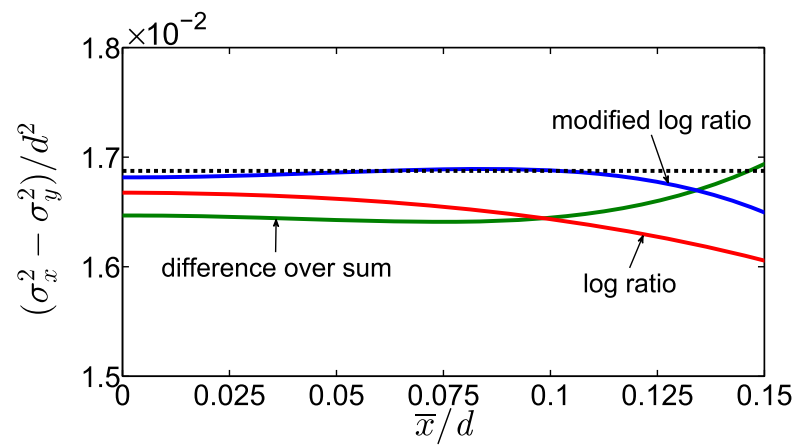

(d) $\bar{y} / d=0.1$

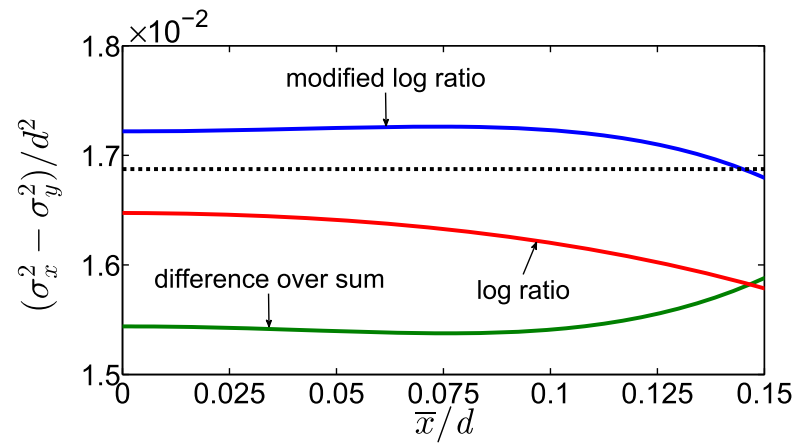

(f) $\bar{y} / d=0.15$

FIG. 9. $\left(\sigma_{x}^{2}-\sigma_{y}^{2}\right) / d^{2}$ as a function of the beam position; $c_{x}=0.1735, c_{y}=0.1885$.

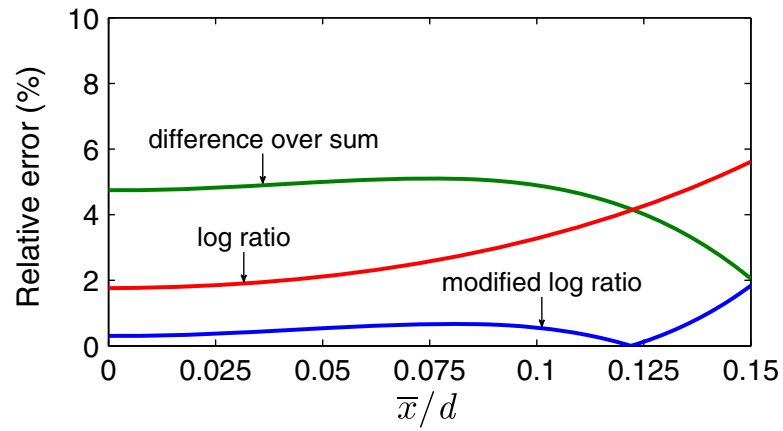

(a) $\bar{y} / d=0.125$

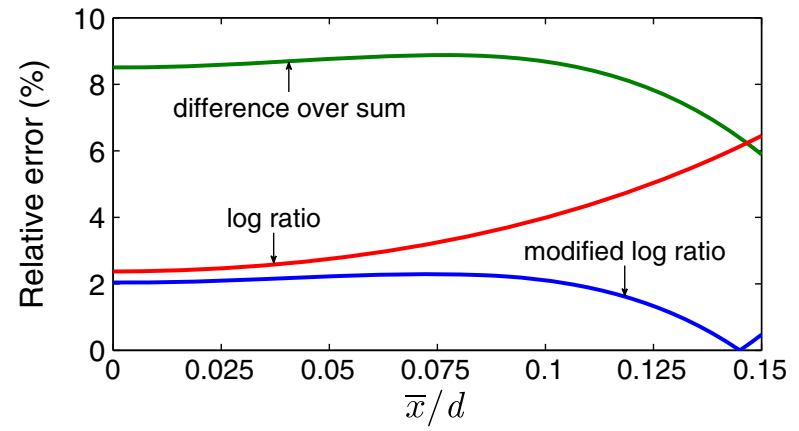

(b) $\bar{y} / d=0.15$

FIG. 10. Absolute value of the relative deviation of the calculated values of $\left(\sigma_{x}^{2}-\sigma_{y}^{2}\right) / d^{2}$ from its ideal value as a function of $\bar{x} / d$ for a fixed value of $\bar{y} / d$. 


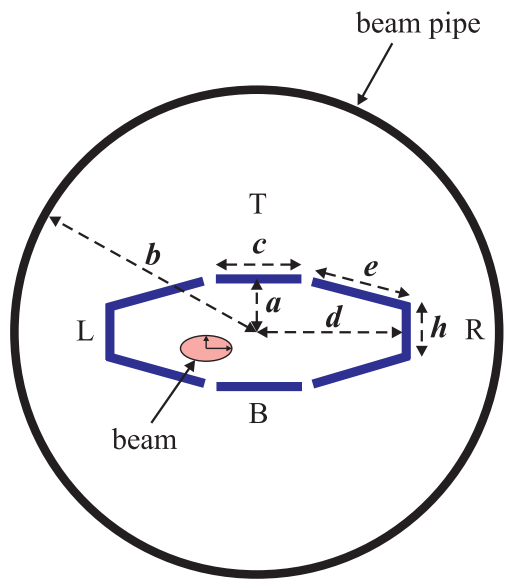

(a)

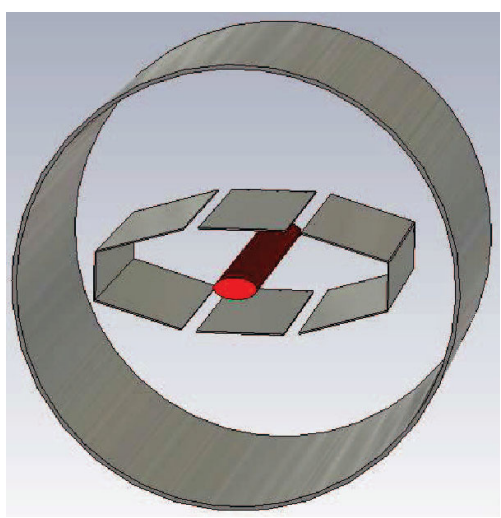

(b)

FIG. 11. (a) Front view of the pickup design installed at GSI SIS-18; $a=35.3 \mathrm{~mm}, b=150 \mathrm{~mm}, c=60 \mathrm{~mm}, d=100.3 \mathrm{~mm}$, $e=62.5 \mathrm{~mm}, h=32 \mathrm{~mm}$, electrode length $l=210 \mathrm{~mm}$, length of the beam pipe $l_{s}=296 \mathrm{~mm}$ [6]. (b) Perspective view of the pickup design with the modeled beam in red, from CST EMS.

entire surface of the modeled beam and the beam pipe was grounded. The quadrupole signal $\Xi$ illustrated in Fig. 12 was obtained using the difference over sum method described in III A,

$$
\Xi=\frac{\left(U_{R}+U_{L}\right)-\left(U_{T}+U_{B}\right)}{U_{R}+U_{L}+U_{T}+U_{B}}
$$

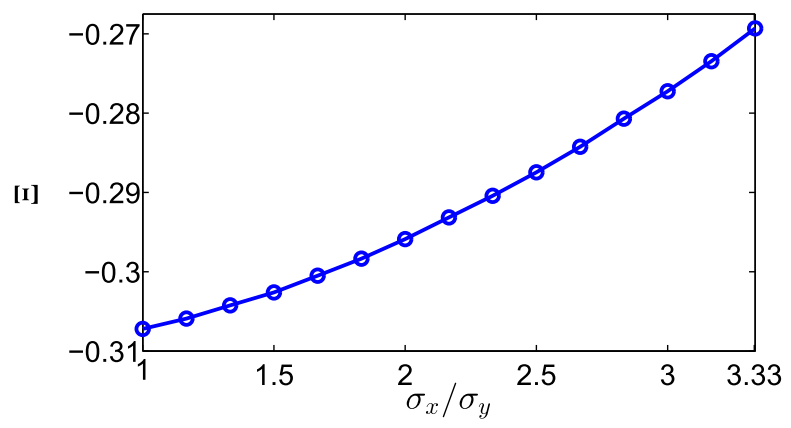

(a)

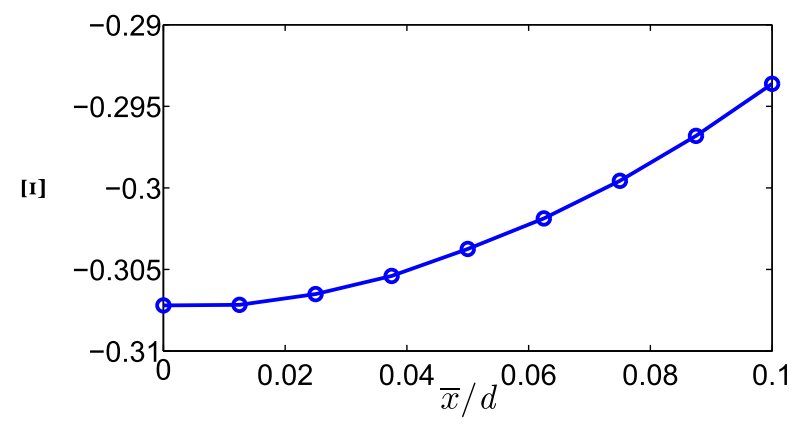

(b)

FIG. 12. (a) Quadrupole signal $\Xi$ for a centered beam as a function of the beam size; (left) as a function of $\sigma_{x} / \sigma_{y}, \sigma_{y}=7.5 \mathrm{~mm}$; (right) as a function of $\sigma_{y} / \sigma_{x}, \sigma_{x}=15 \mathrm{~mm}$. (b) Quadrupole signal $\Xi$ for a round beam $\left(\sigma_{x}=\sigma_{y}=7.5 \mathrm{~mm}\right)$ as a function of the beam position; (left) as a function of $\bar{x} / d, \bar{y}=0$; (right) as a function of $\bar{y} / d, \bar{x}=0$. where $U_{R}, U_{L}, U_{T}$ and $U_{B}$ are the induced voltages on the respective pickup plates. All simulations have been carried out according to the following beam size and beam position values: $7.5 \mathrm{~mm} \leq \sigma_{x} \leq 25 \mathrm{~mm}, \quad 5 \mathrm{~mm} \leq \sigma_{y} \leq 15 \mathrm{~mm}$, $0 \leq \bar{x} \leq 10 \mathrm{~mm}$ and $0 \leq \bar{y} \leq 10 \mathrm{~mm}$. As can be seen in Fig. 12, it is clear that the simulated pickup design is sensitive to both the beam size and the beam position. Hence, it is sensitive to the quadrupole signal according to Eq. (12). The

[I]

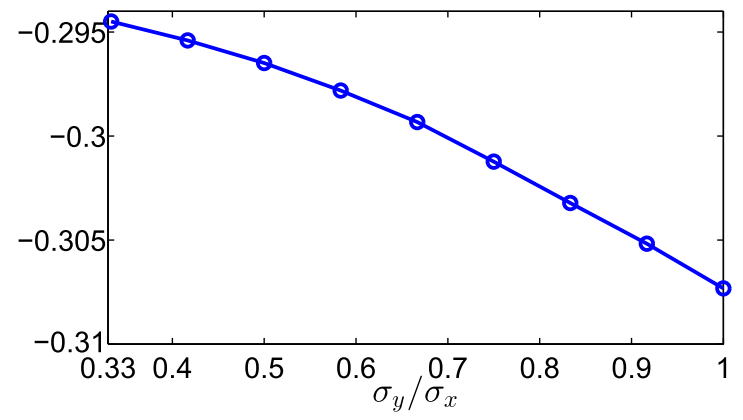

[I]

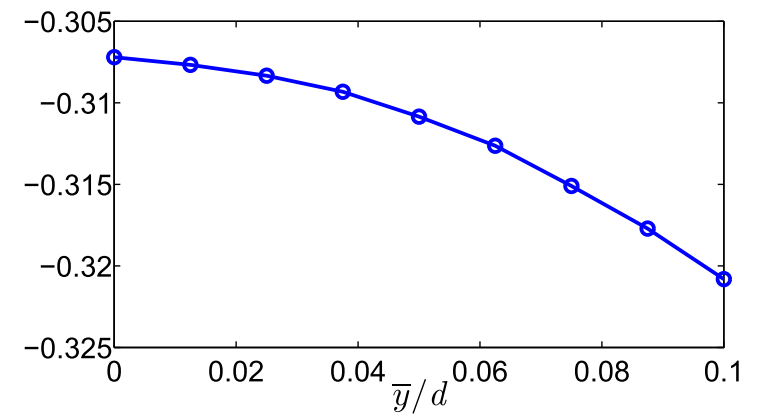

(b) 
pickup sensitivity $S=6.65 \times 10^{-5}\left(1 / \mathrm{mm}^{2}\right)$, which refers to the sensitivity to $\sigma_{x}^{2}-\sigma_{y}^{2}$, was obtained with the left picture in Fig. 12(a) due to its higher number of simulated $\sigma_{x} / \sigma_{y}$ values and using the method of linear regression with the coefficient of determination $R^{2}=0.997$.

The investigated asymmetrical pickup can be characterized as a quadrupole pickup for the used values of the beam size and beam position according to the difference over sum method as

$$
\Xi=S \cdot\left[\left(\sigma_{x}^{2}-\sigma_{y}^{2}\right)+n \cdot\left(\bar{x}^{2}-\bar{y}^{2}\right)\right]+\Xi_{0},
$$

with $n \approx 2$ and $\Xi_{0}=-0.3072$, the value of the quadrupole signal for a round and centered beam. For symmetrical (in both transverse planes) pickup designs, $\Xi_{0}$ is approximatively equal to zero. It should be noted that the sensitivity $S$ is related to the geometrical sizes (radii) of the modeled elliptical beam, which are represented in this case by $\left(\sigma_{x}, \sigma_{y}\right)$ and not to its root mean square (rms).

The characterization of the pickup using the log ratio method can be done analogously to that of the difference over sum.

\section{Numerical determination of $\sigma_{x}^{2}-\sigma_{y}^{2}$}

The modified log ratio method was tested on this asymmetrical pickup model and the results will be compared with those of the traditional signal processing methods. Since the log ratio method is the starting point for the method developed in this paper, let us first look at the quadrupole signal regarding this method, depicted in Fig. 13 as a function of the beam position for the beam dimension $\sigma_{x}=15 \mathrm{~mm}$ and $\sigma_{y}=7.5 \mathrm{~mm}$. Looking at the images in that figure it is clear that in the region of the beam position $\bar{x}$, $\bar{y}$ in less than $5 \%$ of the horizontal pickup radius $b$, the quadrupole signal changes at a maximum of $1 \%$. For this reason, the weighting factors $c_{x}$ and $c_{y}$ for the modified $\log$ ratio have been optimized only in the range of $\bar{x} / d$ and $\bar{y} / d$ from 0.05 to 0.1 . Due to the complicated shape of the pickup electrodes, the determination of $c_{x}$ and $c_{y}$ has been done numerically with the electrostatic solver of CST EMS by using the same approach as with the analytical calculation. The detailed description of this numerical determination of the weighting factors can be found in Appendix A 2. In Fig. 14, the weighting factors for five values of the beam position in the above mentioned range to be optimized are shown. As can be seen in this figure, $c_{x}=0.4719$ and $c_{y}=$ 0.1177 were obtained as the arithmetic mean of all values of the plotted weighting factors, respectively.

The normalized quadrupole moment $\sigma_{x}^{2}-\sigma_{y}^{2} / d^{2}$ for the modified log ratio method can now be obtained according to Eq. (27), after the pickup sensitivity $S$ and the induced signal voltages $U_{R}, U_{T}, U_{L}, U_{B}$ are determined numerically with CST EMS and inserted into the corresponding equations for calculating the quadrupole signal $\Xi_{\bmod }$. However, it should be noted that in the determination of
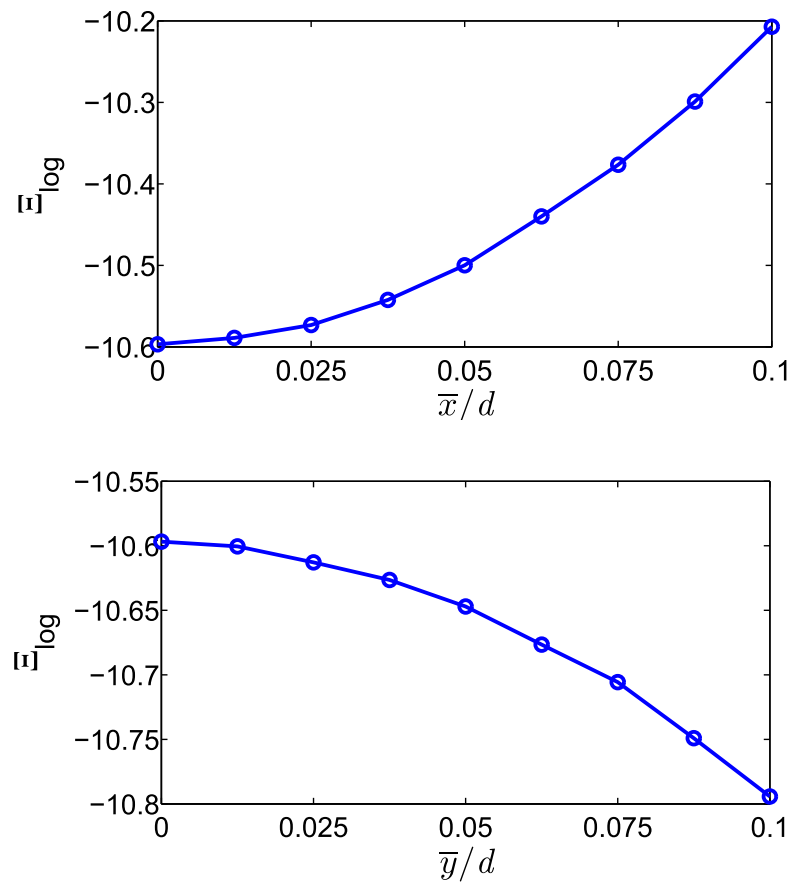

FIG. 13. Quadrupole signal $\Xi_{\log }$ regarding the log ratio method; (above) $\Xi$ as a function of $\bar{x} / d, \bar{y}=0$; (below) $\Xi$ as a function of $\bar{y} / d, \bar{x}=0 ; \sigma_{x}=15 \mathrm{~mm}, \sigma_{y}=7.5 \mathrm{~mm}$.

the quadrupole moment according to Eq. (27), the term $\Xi_{0}$ in Eq. (31) has to be subtracted from the quadrupole signal $\Xi_{\text {mod }}$. The second moment of the beam for the traditional methods is obtained analogously.
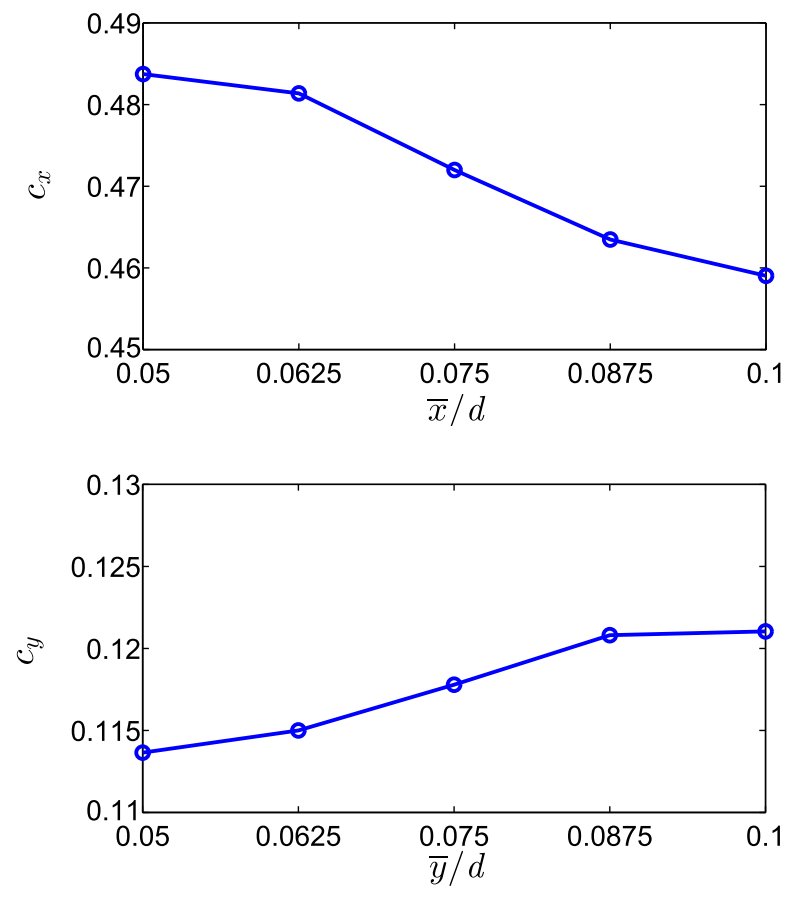

FIG. 14. Weighting factors $c_{x}$ and $c_{y}$ as a function of the beam position; (above) $c_{x}$ as a function of $\bar{x} / d, \bar{y}=0$; (below) $c_{y}$ as a function of $\bar{y} / d, \bar{x}=0 ; \sigma_{x}=15 \mathrm{~mm}, \sigma_{y}=7.5 \mathrm{~mm}$. 


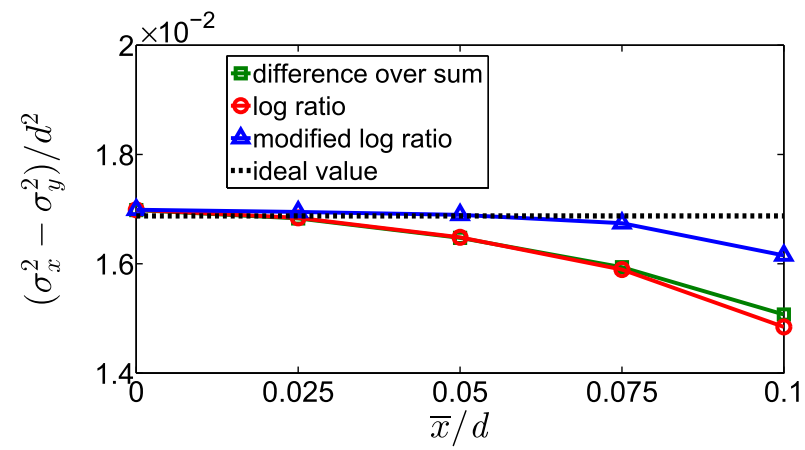

(a) $\bar{y}=0$

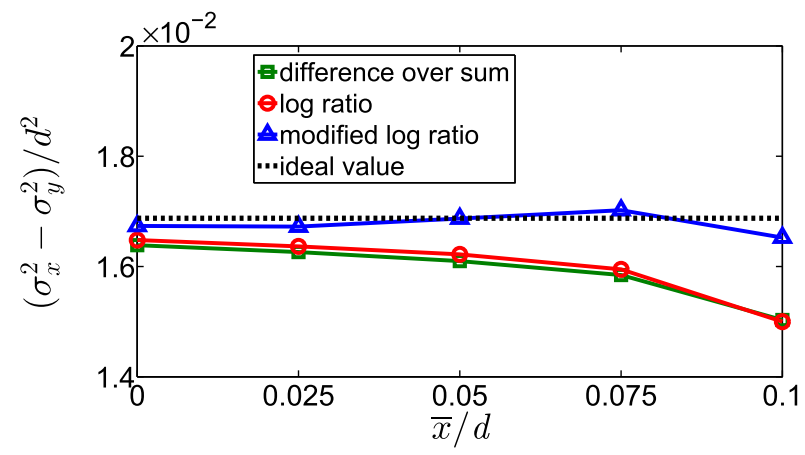

(c) $\bar{y} / d=0.075$

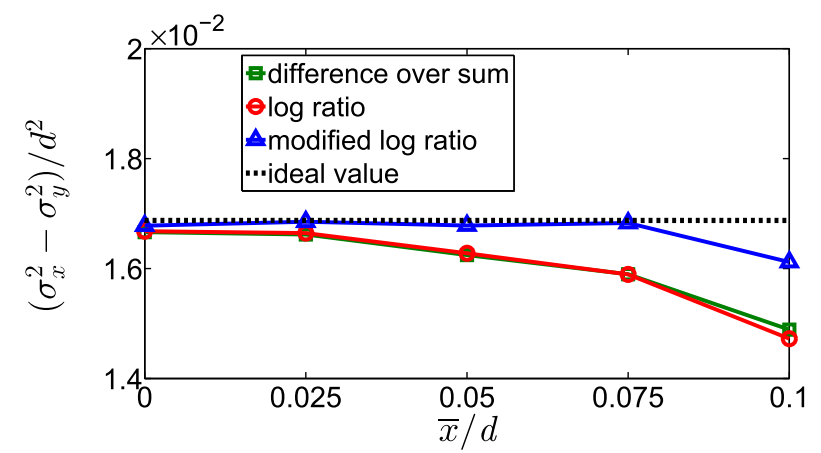

(b) $\bar{y} / d=0.05$

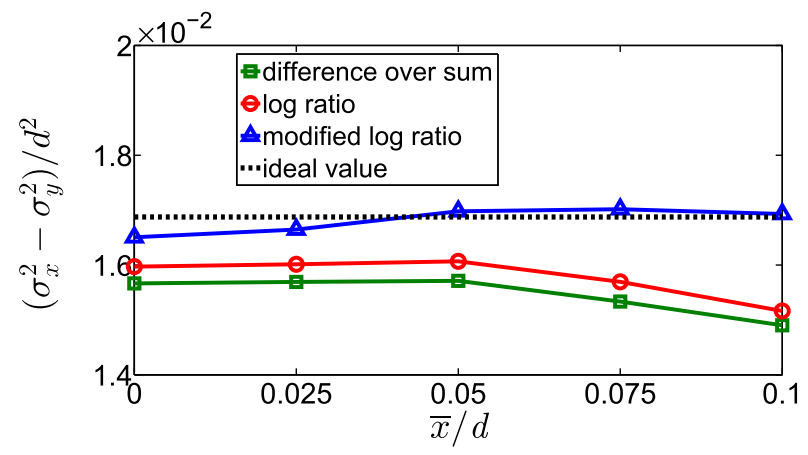

(d) $\bar{y} / d=0.1$

FIG. 15. $\left(\sigma_{x}^{2}-\sigma_{y}^{2}\right) / d^{2}$ as a function of the beam position; $c_{x}=4719, c_{y}=0.1177$.

Figure 15 shows $\sigma_{x}^{2}-\sigma_{y}^{2} / d^{2}$ obtained with the three signal processing methods. The black dotted line in the figure corresponds to the ideal value (setting value) of the quadrupole moment, namely $0.15^{2}-0.075^{2}=$ $1.6875 \times 10^{-2}$. Here, 0.15 is the setting value of $\sigma_{x} / d$ while 0.075 is that of $\sigma_{y} / d$. Looking at the pictures in Fig. 15, it can be seen that for a beam with the dimensions $\left(\sigma_{x}=15 \mathrm{~mm}, \sigma_{y}=7.5 \mathrm{~mm}\right)$ inside of the quadrant bounded by the planes $x=0, x=0.1 d, y=0$ and $y=0.1 d$, the modified log ratio method provides a quadrupole moment with the least dependence on the beam position. To get a better look at the difference between all these methods, the relative deviation of the pickup values of $\left(\sigma_{x}^{2}-\sigma_{y}^{2}\right) / d^{2}$ from its ideal value is depicted in Fig. 16 for the vertical beam position $\bar{y} / d=0.075$ and $\bar{y} / d=0.1$.

The reason for the large deviation in the traditional methods lies, as already shown in analytical investigations, in the dipole signals, since these signals depend not only on the beam position, but also on the sextupole terms. In other words, large values of $\bar{x}$ or $\bar{y}$ lead to a nonlinearity of the dipole signals with respect to the beam position.

The optimization of the modified log ratio method was performed for a fixed beam dimension, namely $\left(\sigma_{x}=15 \mathrm{~mm}, \sigma_{y}=7.5 \mathrm{~mm}\right)$. Accordingly, a general
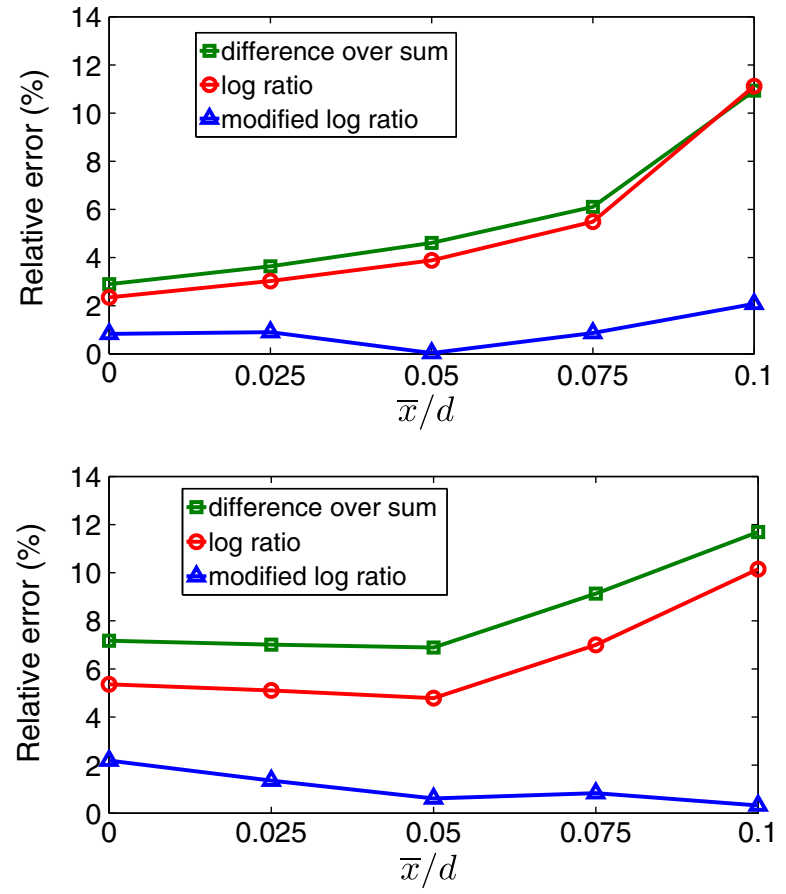

FIG. 16. Absolute value of the relative deviation of the pickup values of $\left(\sigma_{x}^{2}-\sigma_{y}^{2}\right) / d^{2}$ from its ideal value as a function of the beam position; setting beam size: $\sigma_{x}=15 \mathrm{~mm}, \sigma_{y}=7.5 \mathrm{~mm}$; (above) $\bar{y} / d=0.075$, (below) $\bar{y} / d=0.1$. 

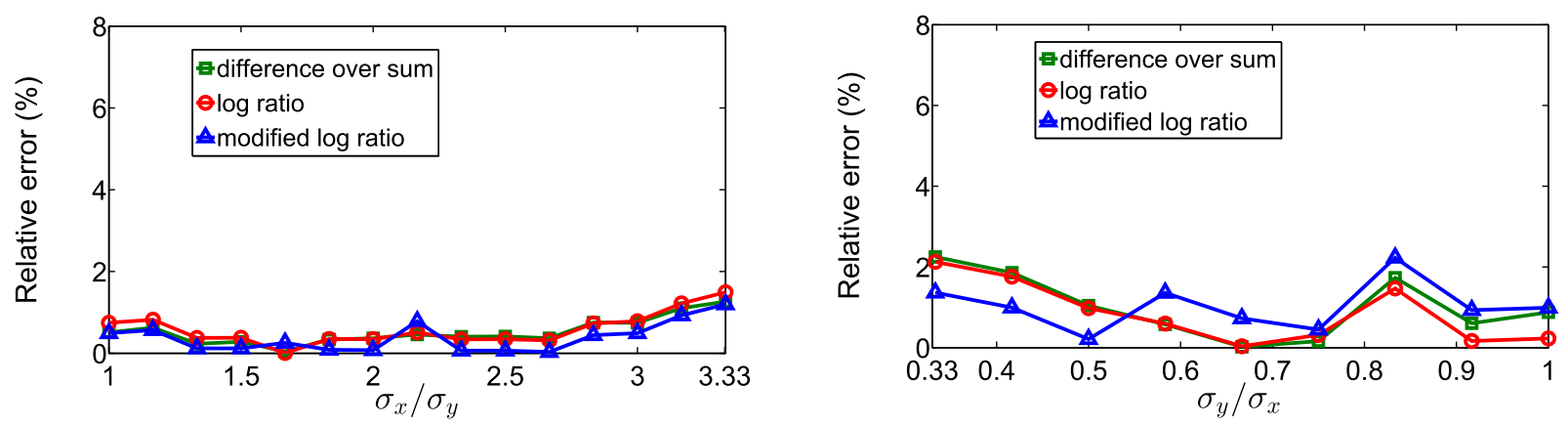

(a) $\bar{x} / d=0.025, \bar{y} / d=0.035$
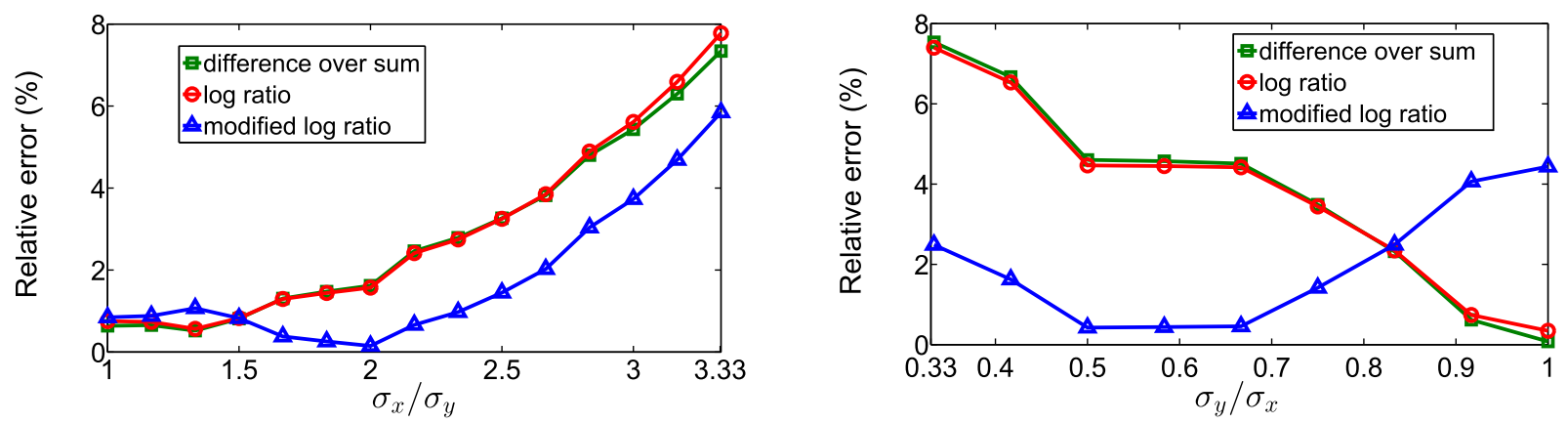

(b) $\bar{x} / d=0.075, \bar{y} / d=0.06$

FIG. 17. Absolute value of the relative error of $\left(\sigma_{x}^{2}-\sigma_{y}^{2}\right) / d^{2}$ from the ideal value: (a) (left) as a function of $\sigma_{x} / \sigma_{y}, \sigma_{y}=7.5 \mathrm{~mm}$; (right) as a function of $\sigma_{y} / \sigma_{x}, \sigma_{x}=15 \mathrm{~mm}$; (b) (left) as a function of $\sigma_{x} / \sigma_{y}, \sigma_{y}=7.5 \mathrm{~mm}$; (right) as a function of $\sigma_{y} / \sigma_{x}, \sigma_{x}=15 \mathrm{~mm}$.

statement about the comparison between the signal processing methods can only be made after the calculation of the quadrupole moment is carried out for different values of the beam size at a fixed beam position, because the term $P$ in Eq. (28) also depends on the beam size. Figure 17 shows the quadrupole moment calculated with all methods for different values of the beam size, while the beam position is held constant. Since the curves of the second moment as a function of the beam size are so close that the difference between them is not clear, Fig. 17 shows only their relative deviations from the ideal value instead. Here, it should be noted that this relative deviation is equal to the absolute deviation divided by the maximum value of the ideal curve. The images in Fig. 17(a) show, due to the relatively small value of the beam position $(\bar{x} / d=0.025$, $\bar{y} / d=0.035$ ), a lower relative deviation from the ideal value as expected.

In the diagrams of Fig. 17(b) in contrast, where $\bar{x} / d=0.075, \bar{y} / d=0.06$, one can see that for almost all used beam dimensions, the modified log ratio method provides the better results.

\section{B. Simulations in the frequency domain}

\section{Cutoff frequency of each electrode}

Since the frequency range of interest for a typical SIS-18 operation is between $10 \mathrm{kHz}$ and $10 \mathrm{MHz}$, a high terminating resistor of $1 \mathrm{M} \Omega$ was connected at the output of each electrode to obtain a low $3 \mathrm{~dB}$ cutoff frequency $f_{c}$ of the plate. However, to get $f_{c}$, the self-capacitance (grounded) of each electrode must be known. This can be found from the capacitance matrix obtained numerically using the electrostatic solver of CST EMS. The pickup used for this calculation is depicted in Fig. 18(a). The different colors of the electrodes in Fig. 18(a) designate the values of the applied potential: (i) right electrode $\mathrm{R}$ (blue): $1 \mathrm{~V}$, (ii) top electrode $\mathrm{T}$ (green): $2 \mathrm{~V}$, (iii) left electrode L (black): $3 \mathrm{~V}$, (iv) bottom electrode $\mathrm{B}$ (red): $4 \mathrm{~V}$, (v) beam pipe: $0 \mathrm{~V}$ (ground). The calculated capacitance matrix with respect to ground is summarized in Table I.

The self-capacitances of the electrodes R, T, L and B can be obtained from the positive values of the diagonal in the capacitance matrix displayed in Table I:

$$
\begin{aligned}
& C_{R}=C_{L}=16.4 \mathrm{pF}, \\
& C_{T}=C_{B}=10.3 \mathrm{pF} .
\end{aligned}
$$

It should be noted that the above self-capacitances of the electrodes have been calculated without taking into account the coaxial line connecting the electrodes with the beampipe and they will be slightly varied depending on the coaxial line dimensions. With the values of the selfcapacitance from Eq. (32) the $3 \mathrm{~dB}$ cutoff frequency of each electrode can be obtained as 


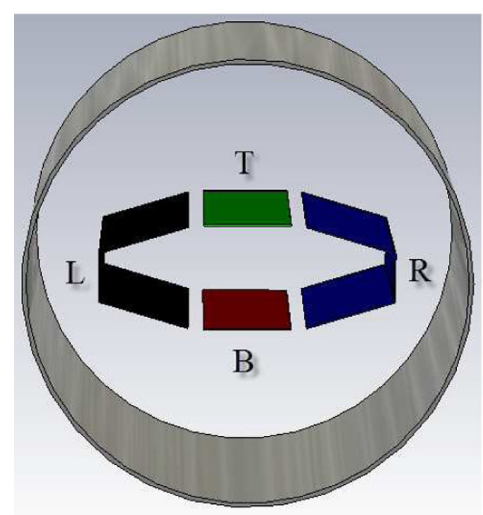

(a)

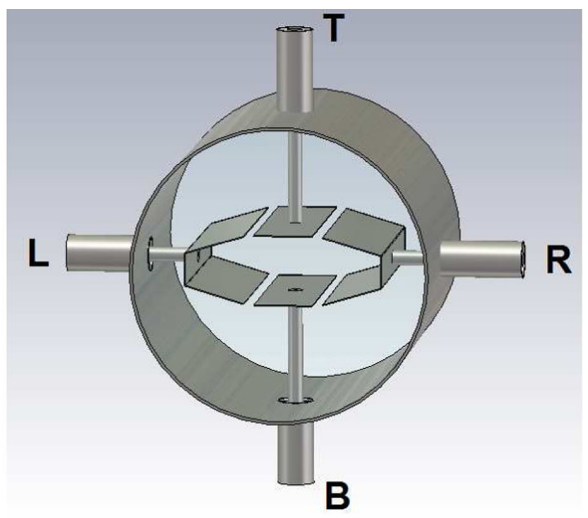

(b)

FIG. 18. (a) Pickup model used for the calculation of the capacitance matrix, from CST EMS. (b) Pickup model used for the simulation in the time domain, from CST PS.

$$
\begin{aligned}
& f_{c, R}=f_{c, L}=\frac{1}{2 \pi R C_{R}}=9.7 \mathrm{kHz}, \\
& f_{c, T}=f_{c,{ }_{B}}=\frac{1}{2 \pi R C_{T}}=15.45 \mathrm{kHz},
\end{aligned}
$$

where $f_{c, R}, f_{c, L}, f_{c, T}$ and $f_{c, B}$ are the cutoff frequency for the right, left, top and bottom electrode, respectively.

From Eq. (33), one can say that using a high terminating resistor of $1 \mathrm{M} \Omega$, almost all frequencies of interest for the SIS-18 operation mentioned above will be passed by the electrodes.

\section{Pickup's sensitivity in the frequency domain}

The pickup design used for the simulations is shown in Fig. 18(b). At the output of each electrode, a high terminating resistor of $1 \mathrm{M} \Omega$ is, as mentioned previously, connected between the inner and outer conductors of the used coaxial transmission line. The simulation was carried out using CST PS, with a PIC solver in the frequency range of $\mathrm{DC} \leq f \leq 200 \mathrm{MHz}$. The quadrupole signal $\Xi(f)$ in the frequency domain obtained regarding the traditional difference over sum method [see Eq. (11)] is illustrated in Fig. 19.

Here $U_{R}, U_{L}, U_{T}$ and $U_{B}$ in Eq. (11) stand for the amplitudes of the fast Fourier transform signal voltages induced on the respective pickup plates. In Fig. 19, one can see that the greater the horizontal beam size $\sigma_{x}$, the greater

TABLE I. Capacitance matrix of the simulated pickup model; values given in $\mathrm{pF}$.

\begin{tabular}{llllc}
\hline \hline & $\mathbf{R}$ & $\mathbf{T}$ & $\mathbf{L}$ & $\mathbf{B}$ \\
\hline $\mathbf{R}$ & 16.4 & -3.06 & -0.178 & -3.06 \\
$\mathbf{T}$ & -3.06 & 10.3 & -3.06 & -0.95 \\
$\mathbf{L}$ & -0.178 & -3.06 & 16.4 & -3.06 \\
$\mathbf{B}$ & -3.06 & -0.95 & -3.06 & 10.3 \\
\hline \hline
\end{tabular}

the quadrupole signal in the whole frequency range. Furthermore, it is obvious that, in the frequency range of interest $(10 \mathrm{kHz}-10 \mathrm{MHz})$ the quadrupole signal is almost constant; the maximum absolute value of the relative deviation of the quadrupole signal in the mentioned frequency range from the value of $\Xi$ at 10 MHZ is below $0.2 \%$.

Now, to get the pickup sensitivity $S(f)$ in the frequency domain, the quadrupole signal for a centered beam at the frequency $1 \mathrm{MHz}$ is depicted in Fig. 20 as a function of the normalized beam size $\sigma_{x} / \sigma_{y}$. Here, the horizontal beam radius $\sigma_{x}$ is varied from 7.5 to $25 \mathrm{~mm}$ while the vertical beam radius $\sigma_{y}$ is $7.5 \mathrm{~mm}$.

From Fig. 20 one can state a good agreement between both the PIC solver of CST PS and the electrostatic solver of CST EMS; the maximum relative deviation of the PIC solver results from the electrostatic ones is below $0.25 \%$. The sensitivity $S=6.57 \times 10^{-5}\left(1 / \mathrm{mm}^{2}\right)$ of the quadrupole pickup to $\sigma_{x}^{2}-\sigma_{y}^{2}$ was determined at the frequency of $1 \mathrm{MHz}$ and, as in the electrostatic case using the method of linear regression with the coefficient of determination $R^{2}=0.9974$, it deviates by approximately $1.1 \%$ from the value of the electrostatic solver $\left[S=6.65 \times 10^{-5}\left(1 / \mathrm{mm}^{2}\right)\right]$.

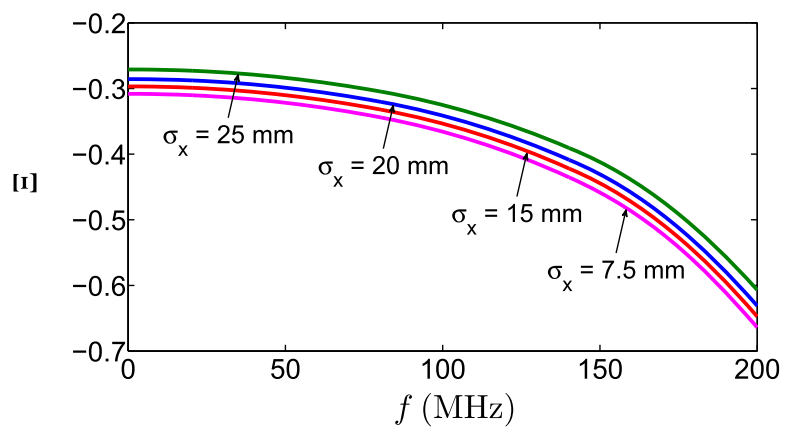

FIG. 19. Quadrupole signal in the frequency domain for different values of the beam size. 


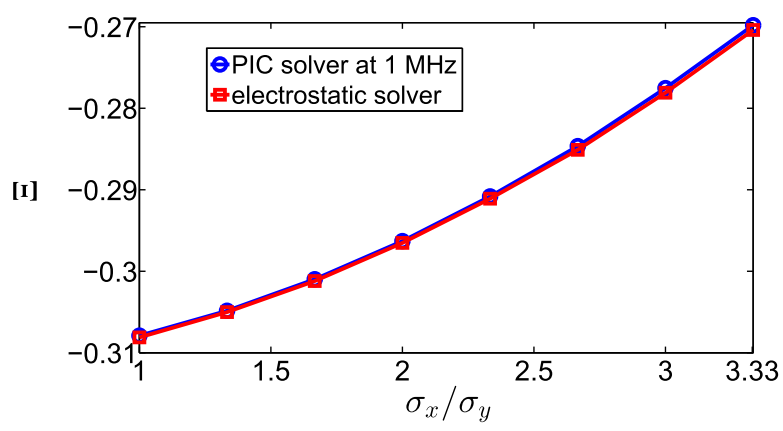

(a)

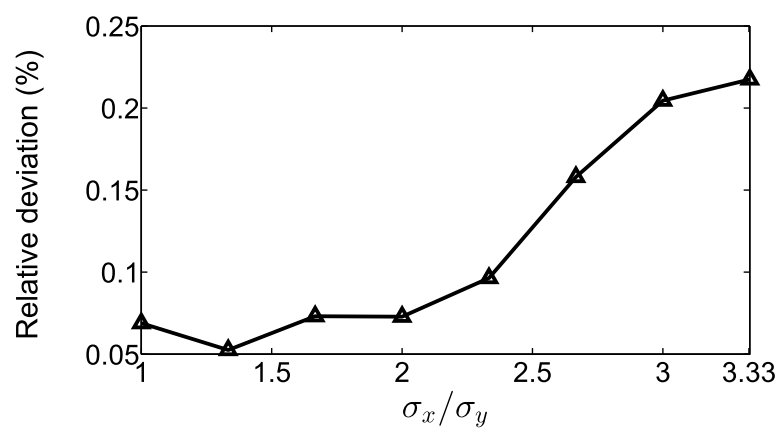

(b)

FIG. 20. (a) $\Xi$ as a function of $\sigma_{x} / \sigma_{y}, \sigma_{y}=7.5 \mathrm{~mm}$; (b) relative deviation of the quadrupole signal obtained with the PIC solver of CST PS from the quadrupole signal obtained with the electrostatic solver of CST EMS.

\section{CONCLUSIONS AND OUTLOOK}

\section{A. Conclusions}

A method to pick up the quadrupole moment of an electrically charged particle beam was developed and presented in this paper. This method was called the modified log ratio.

Analytical and numerical investigations (using the electromagnetic field simulation software CST EMS) on this method were carried out in this work. The results arising from these investigations have shown that the modified log ratio compared with the usual signal processing methods, namely the difference over sum and the log ratio, provides a quadrupole moment $\left(\sigma_{x}^{2}-\sigma_{y}^{2}\right)$ with the least dependence on the beam position. This method was also tested analytically on a magnetic pickup [2], which consists of four symmetrical loop electrodes, by coupling the azimuthal component $B_{\theta}$ of the magnetic field; even with this pickup type, the modified log ratio provides better results than the conventional methods as can be seen in [14] (pages 129 and 130). Due to its reduced dependence on the beam position, the modified $\log$ ratio method can be very suitable in practice to determine the transverse beam emittance using the Miller et al. approach mentioned in the abstract.

In the second part of this paper an asymmetrical BPM installed at GSI SIS-18 was numerically investigated using both the electrostatic solver of CST EMS and the PIC solver of CST PS. The main goal of this study consisted of estimating the properties of this beam position monitor and evaluating its usage as a possible quadrupole pickup. The simulation results have shown that this BPM is sensitive to the used beam sizes and therefore can be used as a quadrupole pickup. Furthermore, there was a good agreement between the two solvers because the maximum relative deviation of the pickup sensitivity to $\sigma_{x}^{2}-\sigma_{y}^{2}$ obtained with the PIC solver from the value of the electrostatic solver was approximately $1.1 \%$. However, the main advantage of the electrostatic solver against the PIC solver is the considerably lower simulation time. Based on these results, one can conclude that, for beams with a longitudinal extent much larger in comparison to the pickup electrode length, the pickup properties can be well determined electrostatically.

After these investigations some measurements have been carried out at GSI SIS-18 with this asymmetrical pickup and it was possible to observe quadrupole signals. This means that the pickup sensitivity obtained numerically in this investigation was sufficiently large that in practice the quadrupole signal could be extracted from the dominant longitudinal signal, which is also called the monopole or sum signal. The results of the mentioned measurements are presented in [15].

\section{B. Outlook}

The method presented in this paper was both analytically and numerically studied with the electromagnetic field simulation software programs mentioned in the previous section. Nevertheless, all output signals at the electrodes were ideally processed, e.g. without consideration of a real differential amplifier with the amplification factor $c$ used in the modified log ratio method (see Fig. 2) as well as external electronic components of the log ratio method, such as the low-frequency filter to pass only frequency components of interest, the transmission cable from the electrodes to the network analyzer. The complete study of the influences that these electronic parts-particularly a real differential amplifier mentioned above-may have on the previous simulation results-in other words, the practical realization of this method-is among the next challenges of this project.

\section{ACKNOWLEDGMENTS}

We would like to thank Peter Forck, Piotr Kowina, Rahul Singh and Marcus Schwickert from GSI Helmholtzzentrum für Schwerionenforschung $\mathrm{GmbH}$ for the provision of simulation models and the measurements carried out with the asymmetrical pickup model installed at GSI SIS-18, which was simulated in this work. Additionally, we would like to thank Wolfgang Ackermann for valuable discussions. 


\section{APPENDIX: DETAILED DERIVATION OF THE MODIFIED LOG RATIO APPROACH}

\section{Quadrupole signal}

$$
\begin{aligned}
\Xi_{\text {mod }}= & 20 \cdot \log \left(\frac{U_{R}^{\text {mod }} \cdot U_{L}^{\text {mod }}}{U_{T}^{\text {mod }} \cdot U_{B}^{\text {mod }}}\right) \\
= & 20 \cdot\left[\log \left(U_{R}^{\text {mod }}\right)+\log \left(U_{L}^{\bmod }\right)-\log \left(U_{T}^{\text {mod }}\right)-\log \left(U_{B}^{\bmod }\right)\right] \\
= & \frac{20}{\ln (10)}\left[4 z_{2}-\left(1+2 c_{x}\right)^{2} z_{1 x}^{2}+\left(1+2 c_{y}\right)^{2} z_{1 y}^{2}-2\left(1+2 c_{x}\right)^{2} z_{1 x} \cdot z_{3 x}-2\left(1+2 c_{y}\right)^{2} z_{1 y} \cdot z_{3 y}\right. \\
& \left.-\left(\frac{\left.\left.\left(1+2 c_{x}\right)^{4} z_{1 x}^{4}-\left(1+2 c_{y}\right)^{4} z_{1 y}^{4}\right)\right]}{2}\right)\right] \\
= & \frac{80}{\ln (10)}\left[z_{2}-\frac{\left(1+2 c_{x}\right)^{2} z_{1 x}^{2}-\left(1+2 c_{y}\right)^{2} z_{1 y}^{2}}{4}\right]+O\left(\frac{1}{d^{4}}, c_{x}, c_{y}\right) \\
\Xi_{\bmod }= & \frac{160}{\ln (10)} \frac{\sin (\alpha)}{\alpha}[\frac{\sigma_{x}^{2}-\sigma_{y}^{2}}{d^{2}}+\underbrace{\left(1-\left(1+2 c_{x}\right)^{2} \frac{\tan (\alpha / 2)}{\alpha}\right)}_{m_{x}} \frac{\bar{x}^{2}}{d^{2}}-\underbrace{\left(1-\left(1+2 c_{y}\right)^{2} \frac{\tan (\alpha / 2)}{\alpha}\right)}_{m_{y}} \frac{\bar{y}^{2}}{d^{2}}] \\
& +O\left(\frac{1}{d^{4}}, c_{x}, c_{y}\right),
\end{aligned}
$$

with

$$
O\left(\frac{1}{d^{4}}, c_{x}, c_{y}\right)=\frac{20}{\ln (10)}\left[-2\left(1+2 c_{x}\right)^{2} z_{1 x} \cdot z_{3 x}-2\left(1+2 c_{y}\right)^{2} z_{1 y} \cdot z_{3 y}-\left(\frac{\left(1+2 c_{x}\right)^{4} z_{1 x}^{4}-\left(1+2 c_{y}\right)^{4} z_{1 y}^{4}}{2}\right)\right]
$$

\section{Numerical calculation of the weighting factors $c_{x}$ and $c_{y}$}

The calculations of $c_{x}$ and $c_{y}$ have been performed, as already mentioned in Sec. IV.A.2 for the beam sizes $\sigma_{x}=15 \mathrm{~mm}, \sigma_{y}=7.5 \mathrm{~mm}$ and for five values of the beam position in the range from 0.05 to 0.1 . Here $d$ is the pickup radius illustrated in Fig. 11. For each value of $\bar{x} / d$, for example, a parameter sweep of $c_{x}$ has been performed with the electrostatic solver of CST EMS until the term $P$ on the right side of Eq. (28) is equal to zero. It should be noted that in the determination of the horizontal factor $c_{x}$, the vertical beam position $\bar{y}$ was set to zero. The values of $c_{x}$ obtained from this parameter sweep are depicted in the following table.

TABLE II. $\quad c_{x}$ values as a function of $\bar{x} / d$.

\begin{tabular}{ll}
\hline \hline $\bar{x} / d$ & $c_{x}$ \\
\hline 0.05 & 0.4837 \\
0.0625 & 0.4814 \\
0.075 & 0.472 \\
0.0875 & 0.4635 \\
0.1 & 0.459 \\
\hline \hline
\end{tabular}

The optimized horizontal weighting factor $c_{x}=0.4719$ was determined as the arithmetic mean of all values of $c_{x}$ in Table II. The vertical damping factor $c_{y}=0.1177$ was obtained analogously.

[1] R. H. Miller, J. E. Clendenin, M. B. James, and J. C. Sheppard, Nonintercepting emittance monitor, in Proceedings of the 12th International Conference on High Energy Accelerators, Batavia, IL, 1983 (Stanford, California, 1983), pp. 602-605.

[2] A. Jansson, Noninvasive measurement of emittance and optical parameters for high-brightness hadron beams in a synchrotron, Ph.D. thesis, Stockholm University, 2001.

[3] M. Chanel, Study of beam envelope oscillations by measuring the beam transfer function with quadrupolar pickup and kicker, PS Division, CERN, Geneva, Switzerland.

[4] S. J. Russell and B. E. Carlsten, Measuring emittance growth due to magnetic bunching of an electron beam using the second moment of its image charge, in Proceedings of the 18th Particle Accelerator Conference, New York, 1999 (IEEE, Los Alamos, New Mexico, 1999), pp. 477-481. 
[5] P. Li, B. G. Sun, Q. Luo, X. H. Wang, H. L. Xu, and P. Lu, New methods of measuring emittance using beam position monitors, in IEEE Nuclear Science Symposium Conference Record, 2007, pp. 1675-1678.

[6] J. Latzko and P. Kowina, Simulations of the position sensitivity for the SIS18 capacitive BPM with a nonstandard geometry, GSI scientific report, 2013.

[7] CST AG, CST EM STUDIO, CST PARTICLE STUDIO, https://www.cst.com/, Bad Nauheimer Straße, Darmstadt, Germany.

[8] V. Chohan, F. Pedersen, S. van der Meer, and D. J. Williams, Measurement of coherent quadrupole oscillations at injection into the antiproton accumulator, in Proceedings of the 2 nd European Particle Accelerator Conference, Nice, France, 1990 (Geneva, Switzerland, 1990), Vol. 1, pp. 320-322.

[9] R. Singh, Tune measurement at GSI SIS-18: Methods and applications, Ph.D. thesis, Technische Universität Darmstadt, 2014.

[10] K. Yanagida, S. Suzuki, and H. Hanaki, Design study of beam position monitors for measuring second-order moments of charged particle beams, Phys. Rev. ST Accel. Beams 15, 012801 (2012).

[11] Heino Henke, Elektromagnetische felder; Theorie und anwendung, Technische Universität Berlin, 2001.

[12] J. Matheson, Nonlinearities in the response of beam position monitors for the LEP spectrometer, Nucl. Instrum. Methods Phys. Res., Sect. A 466, 436 (2001).

[13] F. D. Wells, J. D. Gilpatrick, R. E. Shafer, and R. B. Shurter, Log-ratio circuit for beam position monitoring, in Proceedings of the IEEE 1991 Particle Accelerator Conference (APS Beams Physics) (IEEE, Piscataway, NJ, 1991).

[14] J. A. Tsemo Kamga, Analytische und numerische untersuchung von elektrostatischen quadrupolaren pickups, Ph.D. thesis, Technische Universität Darmstadt, 2015.

[15] R. Singh, P. Forck, P. Kowina, W. F. O. Müller, J. A. Tsemo Kamga, T. Weiland, and M. Gasior, Observations of the quadrupolar oscillations at GSI SIS-18, in Proceedings of IBIC 2014, Monterey, CA, 2014, pp. 629-633. 